\title{
GENERALISED CASTELNUOVO INEQUALITIES
}

\author{
LIAM A. DONOHOE
}

\begin{abstract}
Given a Riemann surface of genus $p$, denoted by $X_{p}$, admitting $j$ linear series of dimension $r$ and degree $n$ Accola derived a polynomial function $f(j, n, r)$ so that $p \leq f(j, n, r)$ and exhibited plane models of Riemann surfaces attaining equality in the inequality. In this paper we provide a classification of all such $X_{p}$ when $r \geq 6$. In addition we classify curves, $X_{p}$, of maximal genus when $X_{p}$ admits two linear series which have a common dimension but different degrees.
\end{abstract}

\section{INTRODUCTION}

In his paper [3] Castelnuovo produced a bound on the genus of an algebraic curve in terms of $n$, the degree, and $r$, the dimension, of a complete, simple, fixed point free, linear series on the curve. He then exhibited the curves which possessed maximal genus. In his paper [1] Accola generalised the idea by allowing the curve to admit several linear series of the same degree and dimension and then set himself the task of answering the questions; is there an upper bound on the genus of the curve in terms of the number of linear series and their degree and dimension and (if it exists) is this bound ever attained? In his paper he produced a bound and then exhibited plane models of curves of maximal genus. We shall call curves of maximal genus generalised Castelnuovo curves. Martens [9], Coppens [5, 6], and Coppens and Kato [7] have considered the problem for the case $r=1$. Coppens gives a classification of Castelnuovo curves possessing several $g_{T}^{1}$ 's in [6]. In this paper we consider the problem for the case $r \geq 6$.

In this section we introduce the arguments used to produce the respective upper bounds and establish some notation and fundamental ideas. All of these are contained in [1]. In $\S 1.1$ and $\S 1.2$ we investigate the nature of the relationship holding between several $g_{n}^{r}$ 's on a generalised Castelnuovo curve. In 1.2.1 we prove that if $g_{n}^{r}$ and $h_{n}^{r}$ are two such linear series then

$$
h_{n}^{r}=g_{n}^{r}+g_{T}^{1}-\mathscr{D}_{1}-\mathscr{D}_{2}
$$

where $g_{T}^{1}$ is a pencil imposing two conditions on $g_{n}^{r}$ and $h_{n}^{r}$ and $\mathscr{D}_{i}$ are fixed divisors. From arguments used originally by Castelnuovo the model for $X_{p}$ in

Received by the editors August 28, 1991 and, in revised form, March 11, 1993.

1991 Mathematics Subject Classification. Primary 14H99; Secondary 30A46.

The author thanks the mathematics department of Brown University for its hospitality during the preparation and revision of this paper. The author also wishes to acknowledge the many helpful suggestions, comments, and corrections made by the referee to the original version of this paper. 
$\mathbf{P}^{r}$ lies on a rational normal surface scroll, the $g_{T}^{1}$ corresponds to the ruling on the scroll and the $\mathscr{D}_{i}$ are the singularities of the model. In $\S 2$ and $\S 3$ we perform the classification proper and we exhibit some plane models of the curves in $\S 4$. Section 5 investigates the situation when $X_{p}$ admits $g_{n}^{r}$ and $h_{n+\rho}^{r}$ and has maximal genus.

Definition 0.0. If $g_{n}^{r}$ and $g_{m}^{s}$ are two complete linear series on a Riemann surface $X_{p}$, with $s \leq r$ and $m \leq n$ we say that $g_{m}^{s}$ imposes $t$ linear conditions on $g_{n}^{r}$ if there is a complete linear series $g_{n-m}^{r-t}$ so that $g_{n}^{r}=g_{m}^{s}+g_{n-m}^{r-t}$. This means that if $\mathscr{D}$ is a general divisor of $g_{m}^{s}$ containing $m$ distinct points then there are $t$ distinct points of $\mathscr{D}, x_{1}, \ldots, x_{t}$ so that $g_{n}^{r}-\left(x_{1}+\cdots+x_{t}\right)$ contains the other points of $\mathscr{D}$ among its fixed points. Also $x_{1}, \ldots, x_{t}$ impose independent conditions on $g_{n}^{r}$, in the following sense: for each $k$ there is a divisor in $g_{n}^{r}$ containing all of the $x_{i}$ except for $x_{k}$.

We intoduce two results dealing with the general position of points on divisors in linear series in projective space.The first one, which may be found in Coolidge $[4$, Theorems 39, 43] is

Theorem 0.1. Let $g_{n}^{r}$ be a simple complete linear series without fixed points. Then the general divisor $\mathscr{D}$ of $g_{n}^{r}$ is made up of $n$ distinct points, and furthermore, any $r$ points of $\mathscr{D}$ impose independent conditions on $g_{n}^{r}$.

The following generalisation of the preceding result is due to Accola [1]. Recall that if $\mathscr{D}$ and $\mathscr{E}$ are two divisors, then their greatest common divisor is denoted by $(\mathscr{D}, \mathscr{E})$.

Theorem 0.2. Suppose that $g_{n}^{r}$ and $g_{m}^{s}$ are two linear series without fixed points so that $r \geq s$. If both series are composite, suppose that there is no involution of which both are compounded. Then, for the general divisor $\mathscr{D}$ in $g_{n}^{r}$ and any divisor $\mathscr{E}$ in $g_{m}^{s}$, the degree of $(\mathscr{D}, \mathscr{E}) \leq s$.

Using these general position theorems we now discuss a notion due to Castelnuovo. Let $g_{n_{1}}^{r_{1}}, \ldots, g_{n_{j}}^{r_{j}}$ be $j$ fixed point free linear series on $X_{p}$. If $g_{n_{1}}^{r_{1}}=g_{n_{i}}^{r_{i}}$ for some $i \geq 2$ then we assume that $g_{n_{1}}^{r_{1}}$ is simple, if all the $g_{n_{i}}^{r_{i}}$ are distinct then we just assume that no two are composed of the same involution. Let $g_{N}^{R}=\sum g_{n_{i}}^{r_{i}}$, and let $g_{N_{1}}^{R_{1}}=g_{N}^{R}+g_{n_{1}}^{r_{1}}$. We obtain a lower bound on $R_{1}$ in the following way. We notice firstly that $R_{1}-R$ corresponds to the number of conditions that $g_{n_{1}}^{r_{1}}$ imposes upon $g_{N_{1}}^{R_{1}}$. Let $t_{i}=r_{i}-1$ if $g_{n_{1}}^{r_{1}}=g_{n_{i}}^{r_{i}}$ and let $t_{i}=\min \left(r_{1}, r_{i}\right)$ otherwise. Then, provided that $n_{1} \geq 1+\sum_{i=1}^{j} t_{i}$, the general position theorem tells us that $g_{n_{1}}^{r_{1}}$ imposes at least $1+\sum_{i=1}^{j} t_{i}$ conditions on $g_{N_{1}}^{R_{1}}$ and we have our lower bound on $R_{1}$.

Using Castelnuovo's method, Accola proves in his paper that if, $r_{1} \geq r_{2} \geq$ $\cdots \geq r_{j}$ and the integers $n_{i}$ and $l_{i}$ satisfy the condition,

$$
n_{i} \geq \sum_{k \geq i}^{j} l_{k} r_{k}-l_{i}+1 \quad \forall i,
$$

then the linear series

$$
W_{0}=l_{1} g_{n_{1}}^{r_{1}}+\cdots+l_{j} g_{n_{j}}^{r_{j}}
$$


has dimension at least

$$
\Psi\left(r_{1}, \ldots, r_{j} ; l_{1}, \ldots, l_{j}\right)=\sum_{i=1}^{j} \frac{\left(l_{i}\right)\left(l_{i}+1\right)}{2} r_{i}-\frac{\left(l_{i}\right)\left(l_{i}-1\right)}{2}+\sum_{k} \sum_{i<k} l_{i} l_{k} r_{k} .
$$

The condition on the $n_{i}$ 's is neccessary because $g_{n_{i}}^{r_{i}}$ cannot impose more than $n_{i}$ conditions on $W_{0}$. An important feature of Accola's estimate of the lower bound on the dimension of $W_{0}$ in the case where $r_{1}=r_{2}=\cdots=r_{j}$, is that it is independent of the order in which the addition takes place.

We turn our attention to the situation where $X_{p}$ admits $j$ simple, complete, special linear series of dimension $r$ and degree $n$. We find a bound on the genus of $X_{p}$ by adding up multiples of the linear series until the resulting linear series is nonspecial (always assuming that Accola's bound is attained, and that Castelnuovo's method may be applied at each stage), then we can use the dimension and degree of the nonspecial linear series to calculate $p$. Curves whose genus attains this upper bound are the subject of this paper. Let us point out that in the case where all the linear series have the same degree and dimension, Accola's formula for the dimension is maximised when $\left|l_{i}-l_{j}\right| \leq 1$ $\forall i, j$; for example, $3 g_{n}^{r}+h_{n}^{r}$ has dimension at least $10 r-3$, while $2 g_{n}^{r}+2 h_{n}^{r}$ has dimension at least $10 r-2$.

This section will introduce several results necessary for our classification of generalised Castelnuovo curves. Unless otherwise stated, we will assume our $g_{n}^{r}$ 's to be complete, special, base point free, and simple. Wherever it appears, the pencil $g_{T}^{1}$ will be assumed to be base point free. Finally we shall assume that $r \geq 6$.

1.1.

Remark 1.1.1. In this section we shall always assume that when we add multiples of two or more linear series together that we can apply Accola's argument to produce a lower bound on the dimension of the resulting linear series. For example, if we add two distinct $g_{n}^{r}$ 's and the resulting linear series has dimension $3 r$, then the condition $n \geq 3 r$ will be understood. The reader is asked to bear this in mind in what follows.

We recall that in Castelnuovo's original argument for a Riemann surface possessing one $g_{n}^{r}$, it was critical for him that the dimension of $2 g_{n}^{r}$ equal $3 r-1$. If $r \neq 5$ it was precisely this fact that allowed him to deduce that the image of the curve $X_{p}$ under the map to $\mathbf{P}^{r}$ defined by $g_{n}^{r}$ lies on a rational normal surface scroll $Y \subset \mathbf{P}^{r}$ [8]. If $r=5$, then the curve may lie on the Veronese surface in $\mathbf{P}^{5}$. Moreover, Castelnuovo was able to deduce the existence of a $g_{T}^{1}$ on the curve cut out by the rulings of the scroll. Finally, since the hyperplanes in $\mathbf{P}^{r}$ cut out $g_{n}^{r}$, he could also deduce that $g_{T}^{1}$ imposes two conditions on $g_{n}^{r}$.

Lemma 1.1.2. If a Riemann surface $X_{p}$ admits two complete linear series $g_{n}^{r}$ and $h_{m}^{r}$, and $r \geq 6$ such that $W=2 g_{n}^{r}+h_{m}^{r}$ has dimension $6 r-1$, the minimal possible, then $X_{p}$ possesses a $g_{T}^{1}$ imposing two conditions on $g_{n}^{r}$.

Proof. Since $W$ has minimal dimension it follows that $2 g_{n}^{r}$ has minimal dimension and the result follows from Castelnuovo's observation. 
We quote, without proof, three results due to Accola [1] that we shall use extensively throughout this paper.

Lemma 1.1.3. Suppose that $g_{n}^{r}$ is simple and $g_{m}^{s}$ is any other linear series, possibly composite, so that $r \geq s$ and $n \geq r+s-1$. Then the dimension of $g_{n}^{r}+g_{m}^{s}$ is at least $r+2 s$.

Lemma 1.1.4. Suppose that $g_{n}^{r}$ and $g_{m}^{s}$ are both simple, are without fixed points, that $n \geq m, r>s$, and that $n \geq 4 s-2$. Suppose further that $s \geq 2$ and that the dimension of $g_{n}^{r}+g_{m}^{s}$ is exactly $r+2 s$, then there are precisely three possibilities for $s$ and $m$ :

(i) $s=2, r=5$, and $g_{n}^{5}=2 g_{m}^{2}$.

(ii) $s=r-2$ and $g_{n}^{r}=g_{m}^{r-2}+g_{n-m}^{1}$.

(iii) $s=r-1$ and $g_{n}^{r}=g_{m}^{r-1}+g_{n-m}^{0}$.

We follow Accola in remarking that if $g_{m}^{r}$ is simple and $g_{m}^{r}+g_{m}^{s}$ has dimension $r+2 s$, and $r \neq 5,3 \leq s \leq r-3$, then $g_{m}^{s}$ is composite. The final result we quote is the following .

Lemma 1.1.5. Suppose $g_{n}^{r}$ is simple and without fixed points, $g_{m}^{s}$ is composite and without fixed points, and that the dimension of $g_{n}^{r}+g_{m}^{s}$ is equal to $r+2 s$. Then, there exists a complete $g_{T}^{1}$, with $s T=m$ so that $g_{m}^{s}=s g_{T}^{1}$. Moreover, $g_{T}^{1}$ imposes precisely two conditions on $g_{n}^{r}$.

The series of results quoted above are foundational to our analysis. In the following two lemmas, we use them to describe completely the nature of $g_{n}^{r}$ when $2 g_{n}^{r}$ has dimension $3 r-1$ and $g_{n}^{r}-g_{T}^{1}$ is composite.

Proposition 1.1.6. Suppose that $X_{p}$ admits a complete, simple linear series $g_{n}^{r}$ and that $2 g_{n}^{r}$ has dimension $3 r-1$. Let $g_{T}^{1}$ be the linear series that imposes two conditions on $g_{n}^{r}$. Then $g_{n}^{r}+\left[g_{n}^{r}-g_{T}^{1}\right]$ has dimension $3 r-4$.

Proof. Note that $g_{T}^{1}$ imposes at least three conditions upon $2 g_{n}^{r}$ and that by assumption, this linear series has dimension $3 r-1$. But $3 r-4$ is the minimum possible dimension for $g_{n}^{r}+\left[g_{n}^{r}-g_{T}^{1}\right]$, so we are done.

Corollary 1.1.7. If $g_{n}^{r}$ is a complete simple linear series so that $2 g_{n}^{r}$ has dimension $3 r-1$ and $g_{n}^{r}-g_{T}^{1}$ is composite (where $g_{T}^{1}$ imposes two conditions on $g_{n}^{r}$ ), then there exists an effective divisor, $\mathscr{D}$ (possibly the zero divisor), so that

$$
g_{n}^{r}=(r-1) g_{T}^{1}+\mathscr{D} .
$$

Proof. By Proposition 1.1.6 and Lemma 1.1.5, we see that $g_{n-T}^{r-2}$ is compounded of a $g_{S}^{1}$ which imposes two conditions on $g_{n}^{r}$, i.e.,

$$
g_{n}^{r}=(r-2) g_{S}^{1}+g_{T}^{1}+\mathscr{D}
$$

where $\mathscr{D}$ is the fixed divisor of $g_{n-T}^{r-2}$. Our task is to show that $g_{S}^{1}=g_{T}^{1}$. Suppose that $g_{T}^{1}$ and $g_{S}^{1}$ are different, then $g_{T}^{1}+g_{S}^{1}$ has dimension at least three. Therefore, rewriting $W=(r-2) g_{S}^{1}+g_{T}^{1}$ as $(r-3) g_{S}^{1}+\left(g_{T}^{1}+g_{S}^{1}\right)$ and using the fact that $g_{S}^{1}$ imposes at least two conditions on $g_{T}^{1}+g_{S}^{1}$ we see that $W$ has dimension at least $3+2(r-3)=2 r-3$. Since $r \geq 6$, this number is greater than $r$ which is a contradiction. Therefore, $g_{T}^{1}=g_{S}^{1}$. 
If $X_{p}$ admits a $g_{n}^{r}$ so that $2 g_{n}^{r}$ has dimension $3 r-1$, then its image in $\mathbf{P}^{r}$ lies on a rational normal surface scroll $Y$. The model will be determined in part by the degree of the $g_{T}^{1}$ which imposes two conditions upon the linear system cut out on $X_{p}$ by hyperplanes. We know of at least one $g_{T}^{1}$ on $X_{p}$, the linear system cut out on image of $X_{p}$ by the lines of the ruling on $Y$. Conceivably, $X_{p}$ could admit many pencils which impose two conditions on $g_{n}^{r}$ and consequently, possess many distinct models. However, using an elementary argument, we can show that there is only one such pencil.

Lemma 1.1.8. If $X_{p}$ admits a complete, simple $g_{n}^{r}$ so that $2 g_{n}^{r}$ has dimension $3 r-1$, then the $g_{T}^{1}$ imposing two conditions upon $g_{n}^{r}$ is unique.

Proof. Suppose that $X_{p}$ admits two distinct pencils, $g_{T}^{1}$ and $g_{S}^{1}$, with both imposing two conditions on $g_{n}^{r}$. Our proof is split into two parts.

(a) Suppose that one of the series $g_{n}^{r}-g_{S}^{1}, g_{n}^{r}-g_{T}^{1}$ is simple, for argument's sake let us assume that the latter series is simple. We have the relationships

$$
g_{n}^{r}=g_{n-S}^{r-2}+g_{S}^{1} \text { and } g_{n}^{r}=g_{n-T}^{r-2}+g_{T}^{1} .
$$

We add these expressions for $g_{n}^{r}$ and compare dimensions. By assumption, $2 g_{n}^{r}$ has dimension $3 r-1$. By Lemma 1.1.3, $g_{n-T}^{r-2}+g_{n-S}^{r-2}$ has dimension $3 r-6+\varepsilon$. Since $g_{T}^{1}$ and $g_{S}^{1}$ are distinct, $g_{T}^{1}+g_{S}^{1}$ has dimension $3+\mu$. Applying 1.1.3 again, the right-hand side has dimension $3 r+\varepsilon+2 \mu+\mu_{1}$, where $\varepsilon, \mu$, and $\mu_{1}$ are all nonnegative. This is a contradiction.

(b) Suppose that both $g_{n}^{r}-g_{T}^{1}$ and $g_{n}^{r}-g_{S}^{1}$ are composite then there exist divisors $\mathscr{P}$ and $\mathscr{Q}$ so that $g_{n}^{r}=(r-1) g_{T}^{1}+\mathscr{P}$ and $g_{n}^{r}=(r-1) g_{S}^{1}+\mathscr{Q}$. We consider

$$
2 g_{n}^{r}=\left(g_{S}^{1}+g_{T}^{1}\right)+(r-2) g_{S}^{1}+(r-2) g_{T}^{1}+\mathscr{Q}+\mathscr{P} .
$$

The linear series $g_{T}^{1}+g_{S}^{1}$ has dimension at least three and both $g_{S}^{1}$ and $g_{T}^{1}$ impose at least two conditions on it therefore, $W=\left(g_{S}^{1}+g_{T}^{1}\right)+(r-2) g_{S}^{1}$ has dimension at least $3+2 r-4=2 r-1$. By a similiar argument $W+(r-2) g_{T}^{1}$ has dimension at least $4 r-5$. Since $r \geq 6$ this contradicts our assumption that $2 g_{n}^{r}$ has dimension $3 r-1$.

Corollary 1.1.9. Suppose that $h_{m}^{r}$ and $g_{n}^{r}$ are two linear series such that $g_{n}^{r}+h_{m}^{r}$ has dimension $3 r$, and both $2 g_{n}^{r}$ and $2 h_{m}^{r}$ have dimension $3 r-1$. Suppose, moreover, that there exist linear series $g_{T}^{1}$ and $g_{S}^{1}$ so that $h_{m}^{r}=(r-1) g_{S}^{1}+\mathscr{P}$ and $g_{n}^{r}=(r-1) g_{T}^{1}+\mathscr{Q}$. Then $g_{T}^{1}=g_{S}^{1}$.

Proof. This follows from the argument given in (ii) above.

Lemma 1.1.10. If $g_{n}^{r}$ and $g_{T}^{1}$ are as in Lemma 1.1.8, then $g_{n}^{r}+g_{T}^{1}$ has dimension $r+2$.

Proof. As in 1.1.7 our proof is split into two parts

(a) Suppose that $g_{n}^{r}-g_{T}^{1}$ is simple, then write $2 g_{n}^{r}=\left[g_{n}^{r}-g_{T}^{1}\right]+\left[g_{n}^{r}+g_{T}^{1}\right]$. If $g_{n}^{r}+g_{T}^{1}$ has dimension $r+3$, then by 1.1 .3 and $1.1 .4,\left[g_{n}^{r}-g_{T}^{1}\right]+\left[g_{n}^{r}+g_{T}^{1}\right]$ has dimension at least $r+3+2(r-2)=3 r-1$. Of course, it has exactly this dimension but then by 1.1 .4 , since $(r+3)-(r-2)=5>3, g_{n}^{r}-g_{T}^{1}$ must be composite. This contradicts our assumption.

(b) Suppose that $g_{n}^{r}-g_{T}^{1}$ is composite, i.e., $g_{n}^{r}=(r-1) g_{T}^{1}+\mathscr{P}$. Suppose that $g_{n}^{r}+g_{T}^{1}$ has dimension $r+3$, then consider the linear series $g_{n}^{r}+(r-1) g_{T}^{1}+\mathscr{P}$. It would have dimension at least $3 r+r-3>3 r-1$, which is a contradiction. 
We reconsider 1.1.8.

Theorem 1.1.11. Suppose that $X_{p}$ is a Riemann surface admitting two linear series, $g_{n}^{r}$ and $h_{m}^{r}$, with (for argument's sake) $m \geq n$ so that both $2 g_{n}^{r}$ and $2 h_{m}^{r}$ have dimension $3 r-1$, and $g_{n}^{r}+h_{m}^{r}$ has dimension $3 r$. Then a $g_{T}^{1}$ which imposes two conditions on $g_{n}^{r}$ also imposes two conditions on $h_{m}^{r}$.

Proof. Our proof splits in the usual way.

(a) Suppose $g_{n}^{r}=(r-1) g_{T}^{1}+\mathscr{P}$. By assumption $h_{m}^{r}+g_{n}^{r}$ has dimension $3 r$; however, if $g_{T}^{1}$ imposes three or more conditions on $h_{m}^{r}$, then $(r-1) g_{T}^{1}+h_{m}^{r}$ has dimension at least $r+3(r-1)=4 r-3>3 r$.

(b) Assume now that $g_{n}^{r}-g_{T}^{1}$ is simple. Since $2 h_{m}^{r}$ has dimension $3 r-1$ there exists a linear series $h_{S}^{1}$ so that $h_{m}^{r}=h_{m-S}^{r-2}+h_{S}^{1}$. We shall show that $h_{S}^{1}=g_{T}^{1}$.

Suppose that $h_{m}^{r}-h_{S}^{1}$ is composite of dimension $r-2$ and that $h_{S}^{1} \neq g_{T}^{1}$. Then, there exists some $\mathscr{P}$ so that $h_{m}^{r}=(r-1) h_{S}^{1}+\mathscr{P}$. Now, $h_{S}^{1}$ must impose three or more conditions on $g_{n}^{r}$. The linear series $W=g_{n}^{r}+(r-1) h_{S}^{1}$ has dimension at least $r+3(r-1)=3 r+(r-3)$. Since $r>3, W$ cannot be contained inside a linear series of dimension $3 r$, which is a contradiction.

We may suppose that $h_{m}^{r}-h_{S}^{1}$ is simple and of dimension $r-2$. Then we can write

$$
g_{n}^{r}+h_{m}^{r}=g_{n-T}^{r-2}+h_{m-S}^{r-2}+\left[g_{T}^{1}+h_{S}^{1}\right] .
$$

If $h_{S}^{1}$ and $g_{T}^{1}$ are distinct, then $h_{S}^{1}+g_{T}^{1}$ has dimension at least three. Since $h_{m-S}^{r-2}$ is simple, the linear series $h_{m-S}^{r-2}+\left[g_{T}^{1}+h_{S}^{1}\right]$ has dimension at least $r+4$. By 1.1.4, the linear series $g_{n-T}^{r-2}+h_{m-S}^{r-2}+\left[g_{T}^{1}+h_{S}^{1}\right]$ has dimension at least $r+4+2(r-2)=3 r$. Of course, by hypothesis, it has exactly this dimension. However, by 1.1 .5 , since $r+4-(r-2)=6>3, g_{n-T}^{r-2}$ must be composite, which contradicts our assumption.

Remark 1.1.12. We note that if $g_{n}^{r}$ is fixed point free and $g_{T}^{1}$ imposes two conditions on $g_{n}^{r}$, so that the linear series $g_{n}^{r}-g_{T}^{1}$ is simple, then $g_{n}^{r}-g_{T}^{1}$ is also fixed point free. If $g_{n}^{r}-g_{T}^{1}=g_{m}^{r-2}+\mathscr{E}$, where $\mathscr{E}$ is effective, then $g_{n}^{r}$ contains the linear series $g_{m}^{r-2}+g_{T}^{1}$ which has dimension $r$. Therefore, $g_{n}^{r}$ contains fixed points, which is a contradiction.

Corollary 1.1.13. Suppose that $g_{n}^{r}$ and $h_{m}^{r}$ are two linear series satisfying the assumptions of 1.1 .11 and that $g_{n}^{r}-g_{T}^{1}$ is simple. Then $W=g_{n}^{r}-g_{T}^{1}+h_{m}^{r}$ has dimension $3 r-3$.

Proof. Since $g_{T}^{1}$ imposes two conditions on both $g_{n}^{r}$ and $h_{m}^{r}, W$ has dimension at most $3 r-3$. If it has dimension $3 r-4$, then since $g_{n}^{r}-g_{T}^{1}$ is simple and fixed point free, we find, by 1.1 .4 , that $h_{m}^{r}=\left[g_{n}^{r}-g_{T}^{1}\right]+h_{S}^{1}$, for some $h_{S}^{1}$. By 1.1.11, we must have $g_{T}^{1}=h_{S}^{1}$, a contradiction.

Lemma 1.1.14 (Accola). Suppose that $g_{n}^{r}$ and $h_{m}^{s}$ are two special linear series, and that $g_{n}^{r}$ is simple. If $g_{n}^{r}+h_{m}^{s}=g_{m+n}^{r+2 s+t}$ where $t \geq 0$ and $r \geq s+1+t$ then $h_{m}^{s}$ imposes no more than $s+t+\mathrm{i}$ conditions on $g_{n}^{r}$.

Proof. Let $\mathscr{F}$ be the fixed point set of $\mathbf{K}-h_{m}^{s}$. Choose a general divisor $\mathscr{E}$ of $h_{m}^{s}$ that corresponds to $m$ distinct points in general position in $\mathbf{P}^{s}$. We may 
assume that $(\mathscr{E}, \mathscr{F})=\varnothing$ and that

$$
\mathscr{E}=x_{1}+\cdots+x_{2 s+t}+x_{2 s+t+1}+\cdots+x_{m}
$$

where $x_{1}+\cdots+x_{2 s+t}$ imposes independent conditions on $g_{n}^{r}+h_{m}^{s}$. There exists a divisor $\mathscr{D}$ in $h_{m}^{s}$, so that $(\mathscr{D}, \mathscr{E})=x_{1}+\cdots+x_{s-1}$. If $\mathscr{L}$ is any divisor in $g_{n}^{r}$ containing $x_{s}+\cdots+x_{2 s+t}$ - recall that $s+t+1 \leq r-$ then $\mathscr{L}$ also contains the points $x_{2 s+t+1}+\cdots+x_{m}$. So $\mathscr{L}$ contains $m-s+1$ points of $\mathscr{E}$. Since $h_{m}^{s}$ is special, $h_{m}^{s}$ imposes $m-s$ conditions on $\mathbf{K}$. Therefore, it cannot impose more conditions on $g_{n}^{r}$ which, also being special, is a subseries of ' $\mathbf{K}$. Therefore, $\mathscr{E} \subset \mathscr{L}$. Thus $h_{m}^{s}$ imposes at most $s+t+1$ conditions on $g_{n}^{r}$.

Corollary 1.1.15. Suppose that $g_{n}^{r}$ and $h_{m}^{r}$ satisfy the hypotheses of 1.1.13. Then $h_{m}^{r}=g_{n-T}^{r-2}+g_{m-n+T}^{0}$.

Proof. Note, as a consequence of 1.1 .11 and 1.1 .13 , that $h_{m}^{r}+g_{n-T}^{r-2}$ has dimension exactly $3 r-3$. By 1.1.14, $g_{n-T}^{r-2}$ imposes at most $r$ conditions on $h_{m}^{r}$, however it cannot impose fewer conditions since if it imposed only $r-1$ conditions, there would be a $g_{m-n+T}^{1}$ which imposed two conditions on $h_{m}^{r}$, which we have seen is impossible. Therefore

$$
h_{m}^{r}=g_{n-T}^{r-2}+g_{m-n+T}^{0} \text {. }
$$

In 1.1.21 we shall extend this result to cover the situation where both $g_{n}^{r}-g_{T}^{1}$ and $h_{m}^{r}-h_{T}^{1}$ are composite.

Remark 1.1.16. Suppose that our Riemann surface admits, in addition to a $g_{n}^{r}$ for which $2 g_{n}^{r}$ has dimension $3 r-1$, a simple $g_{m}^{r-1}$ so that the dimension of $g_{n}^{r}+g_{m}^{r-1}$ equals $3 r-2$. Then by $1.1 .3 g_{m}^{r-1}$ is a subsequence of $g_{n}^{r}$, and since $g_{T}^{1}$ imposes two conditions on $g_{n}^{r}$ it cannot impose more on $g_{m}^{r-1}$.

When we begin to consider the consequences of $X_{p}$ admitting several $g_{n}^{r}$ 's attaining equality in a generalised Castelnuovo inequality we need some information about the dimension of multiples of our $g_{T}^{1}$. Our next result provides this information.

Lemma 1.1.17. Suppose that $X_{p}$ admits $j \geq 3$ linear series of dimension $r \geq 6$, so that $2 g_{i}$ has dimension $3 r-1$ for $1 \leq i \leq j$, and $g_{1}+\cdots+g_{j}$ has minimal dimension. Then the linear series $3 g_{T}^{1}$, where $g_{T}^{1}$ is the pencil imposing two conditions on each $g_{i}$, has dimension three, i.e., it is composite.

Proof. First, we note that if any of the $g_{i}-g_{T}^{1}$ is composite, then 1.1.6 assures us that $(r-1) g_{T}^{1}$ has dimension $r-1$, since $r \geq 6$ our result follows. Therefore, suppose that $g_{i}-g_{T}^{1}$ is simple $\forall i$. Consider the linear series $W=g_{1}+g_{2}+g_{3}$ and rewrite it as

$$
W=\left[g_{1}-g_{T}^{1}\right]+\left[g_{2}-g_{T}^{1}\right]+\left[g_{3}-g_{T}^{1}\right]+3 g_{T}^{1} .
$$

By hypothesis, $W$ has dimension $6 r$, while $W_{1}=W-3 g_{T}^{1}$ has dimension $6 r-12+\varepsilon$. If $3 g_{T}^{1}$ has dimension four, by applying Accola's method, we see that $W$ has dimension at least $6(r-2)+3 \cdot 4+3+1=6 r+4$, which is a contradiction.

Remark 1.1.18. If we have two linear linear series, $g_{n}^{r}$ and $h_{m}^{r}$, such that $2 g_{n}^{r}$ and $2 h_{m}^{r}$ have dimension $3 r-1, g_{n}^{r}+h_{m}^{r}$ has dimension $3 r$, and both $g_{n}^{r}-g_{T}^{1}$ and $h_{m}^{r}-g_{T}^{1}$ are composite, then $\mathscr{Q}$ and $\mathscr{P}$, as exhibited above, cannot be 
empty. If this were the case, and $m=n$, then we would have $g_{n}^{r}=h_{m}^{r}$, while if $m>n, h_{m}^{r}$ would have fixed points.

Theorem 1.1.19. Suppose that we have two linear series $g_{n}^{r}$ and $h_{m}^{r}$, both of dimension $r \geq 6$ and both simple. Suppose that $2 g_{n}^{r}+2 h_{m}^{r}$ has dimension $10 r-$ 2 , the minimum possible (this implies that $2 g_{n}^{r}$ and $2 h_{n}^{r}$ both have dimension $3 r-1$ and that $2 g_{n}^{r}+h_{m}^{r}$ and $2 h_{m}^{r}+g_{n}^{r}$ both have dimension $\left.6 r-1\right)$. Let $g_{T}^{1}$ be as usual, and suppose that at least one of $g_{n}^{r}-g_{T}^{1}$ or $h_{m}^{r}-g_{T}^{1}$ is composite. Then there exist linear series $\hat{g}$ and $\hat{h}$ both of dimension $r+2$, both simple, which satisfy the hypotheses of 1.1 .11 (with $r$ replaced everywhere by $r+2$ ).

Proof. (a) Suppose that $h_{m}^{r}-g_{T}^{1}$ is composite and that $g_{n-T}^{r-2}$ is simple. Let $h_{m}^{r}=(r-1) g_{T}^{1}+\mathscr{Q}$. Let $\hat{g}=g_{n}^{r}+g_{T}^{1}$ and $\hat{h}=h_{m}^{r}+g_{T}^{1}$. Recall that both $\hat{g}$ and $\hat{h}$ have dimension $r+2$ by 1.1 .10 .

(i) We show that $2 \hat{g}$ has dimension $3 r+5$. To see this, first consider the linear series $\hat{g}+g_{n}^{r}$. We claim that it has dimension $3 r+2$. If not, then since $2 g_{n}^{r}$ has dimension $3 r-1$, each time we add a multiple of $g_{T}^{1}$ to $2 g_{n}^{r}$, we increase the dimension by at least four. Rewrite $2 g_{n}^{r}+h_{m}^{r}$ as $g_{n}^{r}+g_{n}^{r}+(r-1) g_{T}^{1}+\mathscr{Q}$ and consider the linear series $g_{n}^{r}+g_{n}^{r}+(r-1) g_{T}^{1}$. It has dimension at least $3 r-1+4(r-1)=6 r-1+(r-4)$, which is a contradiction because $r \geq 6$.

Suppose that $2 \hat{g}$ has dimension $3 r+6$ or more, then every time we add a multiple of $g_{T}^{1}$ to $\hat{g}+g_{n}^{r}$ we increase the dimension by at least four. Rewrite the linear series $2 g_{n}^{r}+h_{m}^{r}$ as $\hat{g}+g_{n}^{r}+(r-2) g_{T}^{1}+\mathscr{Q}$ and then consider the linear series $\hat{g}+g_{n}^{r}+(r-2) g_{T}^{1}$. It has dimension at least $3 r+2+4(r-2)=6 r-1+(r-5)$, which is too big since $r \geq 6$.

(ii) Using the fact that $2 h_{m}^{r}+g_{n}^{r}$ has dimension $6 r-1$ and arguing in a similar way, we can show that $\hat{g}+\hat{h}$ has dimension $3(r+2)$.

(iii) To show that $2 \hat{h}$ has dimension $3(r+2)-1$ we proceed in stages as before. Later $(1.1 .20)$ we shall need the fact that $\hat{h}+h_{m}^{r}$ has dimension $3 r+2$, so we prove it here, along the way, so to speak. We use the fact that $W=2 h_{m}^{r}+g_{n}^{r}$ has dimension $6 r-1$. Rewriting $W$ as $\hat{h}+h+g_{n-T}^{r-2}$, we can compute an upper bound on the number of conditions that $g_{n-T}^{r-2}$ imposes on $W$. We rewrite $W$ again this time as

$$
(r-2) g_{T}^{1}+(r-2) g_{T}^{1}+2 \mathscr{Q}+3 g_{T}^{1}+g_{n-T}^{r-2}
$$

and using Accola's general position theorem we see that $g_{n-T}^{r-2}$ imposes at least $2(r-2)+3+(r-3)+1=3 r-3$ conditions on $W$. This implies that $\hat{h}+h_{m}^{r}$ has dimension no more than $3 r+2$, which is what we wanted to show.

By assumption $W=2 g_{n}^{r}+2 h_{m}^{r}=2 g_{n-T}^{r-2}+2 \hat{h}$ has dimension $10 r-2$ and we shall show that $2 g_{n-T}^{r-2}$ imposes at least $6 r-7$ conditions on $W$. We rewrite

$$
W=2 g_{n-T}^{r-2}+(r-2) g_{T}^{1}+(r-2) g_{T}^{1}+4 g_{T}^{1}+2 \mathscr{Q}=g_{n-T}^{r-2}+W_{1} \text {. }
$$

Then, by applying Accola's general position theorem, $g_{n-T}^{r-2}$ imposes at least $2(r-2)+4+2(r-3)+1=4 r-5$ conditions on $W$ and so $W_{1}$ has dimension no more than $6 r+3$. But $g_{n-T}^{r-2}$ imposes at least $(r-3)+2 r+1$ conditions on $W_{1}$ which implies that $2 \hat{h}$ has dimension no more than $6 r+3-(3 r-2)=3 r+5$. Since it has at least this dimension we are done. 
(b) The case where both $g_{n}^{r}-g_{T}^{1}$ and $h_{m}^{r}-g_{T}^{1}$ are composite is easier, we just mimic the argument given in (i) to complete the proof.

Lemma 1.1.20. Let $g_{n}^{r}$ and $h_{m}^{r}$ be as in 1.1.19. If $g_{n}^{r}-g_{T}^{1}$ is simple and $g_{n}^{r}=g_{m}^{r-1}+\mathscr{D}$, or if $g_{n}^{r}-g_{T}^{1}$ is composite and $g_{n}^{r}=(r-1) g_{T}^{1}+\mathscr{D}$ then $\mathscr{D}$ lies in one divisor of $g_{T}^{1}$.

Proof. (a) Let us consider first the case $g_{n}^{r}=g_{m}^{r-1}+\mathscr{D}$ where $g_{n}^{r}-g_{T}^{1}=g_{n-T}^{r-2}$ is simple. By 1.1.12 $g_{n-T}^{r-2}$ is base point free. We saw in 1.1.6 that $g_{n}^{r}+g_{n-T}^{r-2}$ has dimension $3 r-4$ however, by $1.1 .3 g_{m}^{r-1}+g_{n-T}^{r-2}$ has dimension at least $3 r-5$, since $\mathscr{D} \neq \varnothing$ we see that it has exactly this dimension. Since $g_{n-T}^{r-2}$ is simple and fixed point free we can apply 1.1.4 to see that there exists an effective divisor $\mathscr{E}$ so that

$$
g_{m}^{r-1}=g_{n-T}^{r-2}+\mathscr{E} .
$$

Now we can write

$$
g_{n}^{r}=g_{n-T}^{r-2}+g_{T}^{1}=g_{n-T}^{r-2}+\mathscr{E}+\mathscr{D}
$$

which implies that $g_{T}^{1}-\mathscr{D}$ is effective.

(b) Now we consider the case $g_{n}^{r}=(r-1) g_{T}^{1}+\mathscr{D}$. We want to show that $\mathscr{D}$ imposes just one condition on $\hat{g}$. Then $\hat{g}=g_{m}^{r+1}+\mathscr{D}$ where $g_{m}^{r+1}$ is simple (because it contains $g_{n}^{r}$ ) then we use the fact that $\hat{g}+g_{n}^{r}$ has dimension $3 r+2$ to reduce the argument to the one given in the first case.

The linear series $2 g_{n}^{r}=g_{n}^{r}+(r-1) g_{T}^{1}+\mathscr{D}$ has dimension $3 r-1$. However, the linear series $g_{n}^{r}+(r-1) g_{T}^{1}$ has dimension at least $3 r-2$ since $g_{T}^{1}$ imposes two conditions on $g_{n}^{r}$, if it had dimension $3 r-1$ then $\mathscr{D}$ would be a fixed divisor of $2 g_{n}^{r}$, which we know to be fixed pointfree. Therefore, $\mathscr{D}$ imposes one condition on $2 g_{n}^{r}$ and therefore one condition on $\hat{g}$ which is a subseries of $2 g_{n}^{r}$.

Remark 1.1.21. Suppose that $g_{n}^{r}$ and $h_{m}^{r}$ satisfy the hypotheses of 1.1 .19 and that both $g_{n}^{r}-g_{T}^{1}$ and $h_{m}^{r}-h_{T}^{1}$ are composite. Then, by 1.1.7 and 1.1.11 there are divisors $\mathscr{D}$ and $\mathscr{E}$ so that $g_{n}^{r}=(r-1) g_{T}^{1}+\mathscr{D}$ and $h_{m}^{r}=(r-1) g_{T}^{1}+\mathscr{E}$. By 1.1.20 $\mathscr{D}$ and $\mathscr{E}$ are both contained in a single divisor of $g_{T}^{1}$. If $\mathscr{P}$ is a fixed divisor contained in a single divisor $g_{T}^{1}$ then we denote by $\mathscr{P}$ the divisor $g_{T}^{1}-\mathscr{P}$. We may write

$$
h_{m}^{r}=g_{n}^{r}+g_{T}^{1}-\mathscr{D}-\overline{\mathscr{E}}=\left[g_{n}^{r}-g_{T}^{1}\right]+\overline{\mathscr{D}}+\mathscr{\mathscr { E }},
$$

which extends the result of 1.1.15.

1.2. This section contains the theorems which allow us to describe the nature of the relationship between two linear series on $X_{p}, g_{n}^{r}$ and $h_{m}^{r}$ where $g_{n}^{r}$ and $h_{m}^{r}$ are such that $2 g_{n}^{r}+2 h_{m}^{r}$ has minimal dimension. We suspect a relationship similar to the one described in 1.1.21, our next result confirms our suspicions.

Theorem 1.2.1. Suppose that $g_{n}^{r}$ and $h_{m}^{r}$ are two linear series on $X_{p}$ so that $2 g_{n}^{r}+2 h_{m}^{r}$ has minimal dimension $10 r-2$. Then there exist effective divisors $\mathscr{D}_{i}, i=1,2$ so that

$$
h_{m}^{r}=g_{n}^{r}+g_{T}^{1}-\mathscr{D}_{1}-\mathscr{D}_{2},
$$


and there are two possibilities:

(i) if $\left(\mathscr{D}_{1}, \mathscr{D}_{2}\right)=\varnothing$ then $\mathscr{D}_{1}$ and $\mathscr{D}_{2}$ are fuxed divisors which are contained in different divisors of $g_{T}^{1}$.

(ii) If $\left(\mathscr{D}_{1}, \mathscr{D}_{2}\right) \neq \varnothing$ then $\mathscr{D}_{1}$ and $\mathscr{D}_{2}$ are contained in the same divisor of $g_{T}^{1}$, but $\mathscr{D}_{1}+\mathscr{D}_{2}$ is not contained in any divisor of $g_{T}^{1}$.

The first of these situations we shall describe as generic, the second we shall call nongeneric.

Proof. Note that if both $g_{n}^{r}-g_{T}^{1}$ and $h_{m}^{r}-g_{T}^{1}$ are composite then the result follows from 1.1.21. We may as well assume therefore that $g_{n}^{r}-g_{T}^{1}$ is simple.Consider the linear series $h_{m}^{r}+g_{n}^{r}-g_{T}^{1}$. We saw above, in 1.1 .14 , that it has dimension $3 r-3$, and that as a consequence of this

$$
h_{m}^{r}=g_{n-T}^{r-2}+g_{m-n+T}^{0} .
$$

Let us examine the linear series $h_{m}^{r}+\left[g_{n}^{r}+g_{T}^{1}\right]$, we claim it has dimension $3 r+3$. If either $g_{n}^{r}-g_{T}^{1}$ or $h_{m}^{r}-g_{T}^{1}$ is composite, then, using the arguments of 1.1 .19 , we see immediately that $\hat{g}+\hat{h}+g_{T}^{1}$ has dimension $3(r+2)+3$. So we may assume that $h_{m}^{r}-g_{T}^{1}$ is simple. We establish first that $3 r+3$ is a lower bound. By 1.1.10 $g_{n}^{r}+g_{T}^{1}$ has dimension $r+2$, by $1.1 .3\left[g_{n}^{r}+g_{T}^{1}\right]+h_{m}^{r}$ has dimension at least $3 r+2$. If it has dimension exactly $3 r+2$ then by 1.1.4 there exists a $g_{n-m+T}^{1}$ so that $g_{n}^{r}+g_{T}^{1}=h_{m}^{r}+g_{n-m+T}^{1}$. Consequently $g_{n-m+T}^{1}$ imposes two conditions on the series $g_{n}^{r}$; if $m>n$ this contradicts 1.1.8, since $g_{T}^{1}$ has no fixed points. If $n=m$ then by 1.1 .8 we conclude that $g_{n}^{r}=h_{n}^{r}$ also a contradiction. To see that $3 r+3$ is also an upper bound, we consider the linear series $W=2 g_{n}^{r}+h_{m}^{r}$. By assumption, $W$ has dimension $6 r-1$. Rewrite it as $\left[g_{n}^{r}-g_{T}^{1}\right]+\left[g_{n}^{r}+h_{n}^{r}+g_{T}^{1}\right]$. Now we rewrite it again, this time as $2\left[g_{n}^{r}-g_{T}^{1}\right]+\left[h_{m}^{r}-g_{T}^{1}\right]+3 g_{T}^{1}$. By Accola's count $g_{n}^{r}-g_{T}^{1}$ imposes at least $2(r-3)+(r-2)+3+1=3 r-4$ conditions on $W$. The claim follows immediately.

By $1.1 .14, h_{m}^{r}$ imposes at most $r+2$ conditions on $g_{n}^{r}+g_{T}^{1}$. By Accola's general position theorem $h_{m}^{r}$ imposes at least $r+1$ conditions on $g_{n}^{r}+g_{T}^{1}$. If it impose exactly $r+1$ conditions, then $X_{p}$ admits a $g_{n-m+T}^{1}$ imposing two conditions on both $h_{m}^{r}$ and $g_{n}^{r}$, which is impossible for the reasons given above. Therefore $h_{m}^{r}$ imposes two conditions on $g_{n}^{r}+g_{T}^{1}$ and there exists an effective divisor $g_{n-m+T}^{0}$ so that

$$
h_{m}^{r}+g_{n-m+T}^{0}=g_{n}^{r}+g_{T}^{1} .
$$

We also have

$$
h_{m}^{r}+g_{n-m+T}^{0}=g_{n-T}^{r-2}+2 g_{T}^{1}
$$

Therefore,

$$
h_{m}^{r}=g_{n-T}^{r-2}+\left[2 g_{T}^{1}-g_{n-m+T}^{0}\right] .
$$

Combining (1.1) and (1.2), we find that $g_{m-n+T}^{0}$ and $g_{n-m+T}^{0}$ are both contained in $2 g_{T}^{1}$ and that

$$
2 g_{T}^{1}=g_{n-m+T}^{0}+g_{m-n+T}^{0} .
$$


Using an argument similar to the one given in 1.1 .17 we can show that $2 g_{T}^{1}$ has dimension two. So we may write

$$
2 g_{T}^{1}-g_{m-n+T}^{0}=g_{n-m+T}^{0} .
$$

Clearly, neither $g_{m-n+T}^{0}$ nor $g_{n-m+T}^{0}$ can lie in a single divisor of $g_{T}^{1}$. Therefore, there exist divisors $\mathscr{E}_{1}$ and $\mathscr{E}_{2}$ of degree $s$ and $q$ respectively, where $T+m-n=s+q$, so that

$$
g_{n-m+T}^{0}=\left[g_{T}^{1}-\mathscr{E}_{1}\right]+\left[g_{T}^{1}-\mathscr{E}_{2}\right] .
$$

Then, letting $\mathscr{D}_{1}=g_{T}^{1}-\mathscr{E}_{1}$ and $\mathscr{D}_{2}=g_{T}^{1}-\mathscr{E}_{2}$, we obtain

$$
h_{m}^{r}=g_{n}^{r}+g_{T}^{1}-\mathscr{D}_{1}-\mathscr{D}_{2} \text {, }
$$

and either

(i) $\mathscr{D}_{1}$ and $\mathscr{D}_{2}$ lie in different divisors of $g_{T}^{1}$,

(ii) $\mathscr{D}_{1}$ and $\mathscr{D}_{2}$ lie in the same divisor of $g_{T}^{1}$.

Note that if (ii) is the case, then we cannot have $\left(\mathscr{D}_{1}, \mathscr{D}_{2}\right)=\varnothing$. If $m \geq n$ then $g_{T}^{1}-\mathscr{D}_{1}-\mathscr{D}_{2}$ would be an effective divisor and $h_{m}^{r}$ would have fixed points, while if $m=n$, we would have $h_{n}^{r}=g_{n}^{r}$. Both of these conclusions are contradictory to our assumptions.

Lemma 1.2.2. If we are in the generic situation above then (i) $\mathscr{D}_{i}$ imposes one condition on $g_{n}^{r}$, and (ii) $g_{n}^{r}-\mathscr{D}_{i}$ is fixed point free for each $i$.

Proof. If $\mathscr{D}$ is contained in a single divisor of $g_{T}^{1}$, let us denote the divisor $g_{T}^{1}-\mathscr{D}$ by $\bar{D}$. Let $s$ and $q$ be the degrees of $\mathscr{D}_{1}$ and $\mathscr{D}_{2}$, respectively. Let us suppose that $g_{n}^{r}-g_{T}^{1}$ and $h_{m}^{r}-g_{T}^{1}$ are both simple, if this is not the case then we can replace $g_{n}^{r}$ and $h_{m}^{r}$ in (1.3) above by $\hat{g}$ and $\hat{h}$, both of which will then satisfy the hypotheses of 1.1 .11 , which are the only ones we really need in this proof.

(i) Suppose that $\mathscr{D}_{1}$ imposes two conditions on $g_{n}^{r}$. Let $g_{n-s}^{r-2}=g_{n}^{r}-\mathscr{D}_{1}$ and let $g_{n-T}^{r-2}=g_{n}^{r}-g_{T}^{1}$, by assumption the latter is simple and fixed point free. Then

$$
h_{m}^{r}=g_{n}^{r}+g_{T}^{1}-\mathscr{D}_{1}-\mathscr{D}_{2}=\left[g_{n}^{r}-\mathscr{D}_{1}\right]+\left[g_{T}^{1}-\mathscr{D}_{2}\right] .
$$

But, since $\mathscr{D}_{1}$ is contained in a single divisor of $g_{T}^{1}$,

Therefore

$$
g_{n}^{r}-\mathscr{D}_{1}=g_{n-T}^{r-2}+\overline{\mathscr{D}_{1}}=g_{n-s}^{r-2} \text {. }
$$

$$
g_{n-s}^{r-2}=\left[h_{m}^{r}-g_{T}^{1}\right]+\mathscr{D}_{2}=h_{m-T}^{r-2}+\mathscr{D}_{2}
$$

and we conclude that $\left(\overline{\mathscr{D}}_{1}, \mathscr{D}_{2}\right) \neq \varnothing$, which contradicts our assumption that the $\mathscr{D}_{i}$ lie in different divisors of $g_{T}^{1}$. Therefore, $g_{n}^{r}-\mathscr{D}_{i}$ has dimension one for each $i$.

(ii) Suppose that $g_{n}^{r}-\mathscr{D}_{1}=g_{m}^{r-1}+\mathscr{F}$ where $\mathscr{F} \neq \varnothing$, since $g_{m}^{r-1}$ contains $g_{n-T}^{r-2}$ it is simple. By $1.1 .20,(\mathrm{i}), \mathscr{F}+\mathscr{D}_{1}$ must lie in one divisor of $g_{T}^{1}$. Then

$$
h_{m}^{r}=g_{m}^{r-1}+\mathscr{F}+\overline{\mathscr{D}_{2}}
$$

again, by 1.1.20 we see that $\mathscr{F}+\overline{\mathscr{D}_{2}}$ must lie in one divisor of $g_{T}^{1}$, a contradiction. Note also that $h_{m}^{r}-\overline{\mathscr{D}}_{i}$ is fixed point free and of dimension $r-1$.

We would like to make the same type of claim for the nongeneric case. Of course, we cannot completely duplicate the last result, but the following lemma introduces some structure into this situation. 
Lemma 1.2.3. If $g_{n}^{r}$ and $h_{m}^{r}$ are as in 1.2.1 and

$$
h_{m}^{r}=g_{n}^{r}+g_{T}^{1}-\hat{\mathscr{D}}_{1}-\hat{\mathscr{D}}_{2} \text { with }\left(\hat{\mathscr{D}}_{1}, \hat{\mathscr{D}}_{2}\right) \neq \varnothing,
$$

we shall prove that it is possible to find $\mathscr{D}_{1}$ and $\mathscr{D}_{2}$ so that $\mathscr{D}_{1}+\mathscr{D}_{2}=\hat{\mathscr{D}}_{1}+\hat{\mathscr{D}}_{2}$ and

(i) $\left(\mathscr{D}_{1}, \mathscr{D}_{2}\right)=\mathscr{D}_{2}$.

(ii) $\mathscr{D}_{1}$ imposes one condition on $g_{n}^{r}$.

(iii) $g_{n}^{r}-\mathscr{D}_{1}$ has no fixed points.

(iv) $\left(\mathscr{D}_{2}, \overline{\mathscr{D}_{1}}\right)=\varnothing$.

Proof. Let us suppose that $g_{n}^{r}-g_{T}^{1}$ and $h_{m}^{r}-g_{T}^{1}$ are both simple. If this is not the case then we can replace $g_{n}^{r}$ and $h_{m}^{r}$ in (1.3) above by $\hat{g}$ and $\hat{h}$, both of which will then satisfy the hypotheses of 1.1.11. The proof is constructive. Suppose that

$$
h_{m}^{r}=g_{n}^{r}+g_{T}^{1}-\hat{\mathscr{D}}_{1}-\hat{\mathscr{D}}_{2}
$$

where $\left(\hat{\mathscr{D}}_{1}, \hat{\mathscr{D}}_{2}\right) \neq \varnothing$.

(i) Let $\mathscr{B}=\left(\hat{\mathscr{D}}_{1}, \hat{\mathscr{D}}_{2}\right)$. Then we can write $\hat{D}_{1}=\mathscr{A}+\mathscr{B}$ and $\hat{\mathscr{D}}_{2}=\mathscr{C}+\mathscr{B}$ where $(\mathscr{A}, \mathscr{C})=\varnothing$. Let us write

$$
g_{T}^{1}=\mathscr{A}+\mathscr{B}+\mathscr{C}+\mathscr{E} .
$$

Then we have

$$
h_{m}^{r}=g_{n}^{r}+g_{T}^{1}-(\mathscr{A}+\mathscr{B}+\mathscr{C})-\mathscr{B} .
$$

Clearly, if we let $\tilde{\mathscr{D}}_{1}=\mathscr{A}+\mathscr{B}+\mathscr{C}$ and $\tilde{\mathscr{D}}_{2}=\mathscr{B}$, we have a pair of divisors satisfying (i).

(ii) Next, we must show that $\tilde{\mathscr{D}}_{1}$ imposes only one condition on $g_{n}^{r}$. Suppose that it does not. Note that

$$
h_{m}^{r}-g_{T}^{1}=\left(g_{n}^{r}-\tilde{\mathscr{D}}_{1}\right)-\tilde{\mathscr{D}}_{2} .
$$

Let $\mathscr{E}=g_{T}^{1}-\tilde{D}_{1}$. If $\tilde{D}_{1}$ imposes two conditions on $g_{n}^{r}$, then since $g_{T}^{1}$ itself imposes two conditions on $g_{n}^{r}$, we must have

$$
h_{m}^{r}-g_{T}^{1}+\tilde{\mathscr{D}}_{2}=\left(g_{n}^{r}-g_{T}^{1}\right)+\mathscr{E} .
$$

Both sides have dimension $r-2$ and $\mathscr{E}$ is the fixed point set of the right-hand side. Clearly, $\tilde{\mathscr{D}}_{2}$ and $\mathscr{E}$ have the same degree, but they cannot be equal, since $g_{n}^{r}$ and $h_{m}^{r}$ are distinct. Thus, we have a contradiction.

(iii) Suppose that $g_{n}^{r}-\tilde{\mathscr{D}}_{1}=g_{1}^{r-1}+\hat{\mathscr{E}}$, where $g_{1}^{r-1}$ has dimension $r-1$ and $\hat{\mathscr{E}}$ is an effective divisor, possibly of degree zero. Assuming that $\hat{\mathscr{E}} \neq 0$, we proceed as follows, let $g_{T}^{1}=\mathscr{A}+\tilde{\mathscr{D}}_{2}+\mathscr{C}+\mathscr{E}$ where $\tilde{\mathscr{D}}_{1}=\mathscr{A}+\tilde{\mathscr{D}}_{2}+\mathscr{C}$, then

$$
h_{m}^{r}=\left(g_{n}^{r}-\tilde{\mathscr{D}}_{1}\right)+\left(g_{T}^{1}-\tilde{\mathscr{D}}_{2}\right)=g_{1}^{r-1}+\mathscr{A}+\mathscr{C}+\mathscr{E}+\hat{\mathscr{E}}
$$

and

$$
g_{n}^{r}=g_{1}^{r-1}+\tilde{\mathscr{D}}_{1}+\hat{\mathscr{E}} .
$$

Adding the expressions for $g_{n}^{r}$ and $h_{m}^{r}$, we obtain a linear series of dimension $3 r$. Since $g_{T}^{1}=\mathscr{A}+\tilde{\mathscr{D}}_{2}+\mathscr{C}+\mathscr{E}$, we find that

$$
g_{n}^{r}+h_{m}^{r}=\left(g_{1}^{r-1}+g_{T}^{1}\right)+g_{1}^{r-1}+(\mathscr{A}+\mathscr{C}+2 \hat{\mathscr{E}}) .
$$


Because $g_{n}^{r}-g_{T}^{1}$ is simple, it follows that $g_{1}^{r-1}$ is also simple, therefore the linear series $g_{1}^{r-1}+g_{T}^{1}$ has dimension at least $r+1$. By Accola's count, the linear series $\left(g_{1}^{r-1}+g_{T}^{1}\right)+g_{1}^{r-1}$ has dimension at least $3 r-1$. Clearly, its dimension can be no greater than this - since $\hat{\mathscr{E}} \neq \varnothing$, and $g_{n}^{r}+h_{m}^{r}$ is fixed point free - so we may conclude that the divisor $\mathscr{A}+\mathscr{E}+2 \hat{\mathscr{E}}$ imposes one condition on the linear series $g_{n}^{r}+h_{m}^{r}$. Therefore, it imposes one condition on $g_{n}^{r}$ and we may deduce that there exists some effective divisor $\mathscr{F}$ such that

$$
\mathscr{A}+\tilde{\mathscr{D}}_{2}+\mathscr{C}+\hat{\mathscr{E}}=\mathscr{A}+\mathscr{C}+2 \hat{\mathscr{E}}+\mathscr{F} \text {. }
$$

So $\left(\tilde{\mathscr{D}}_{2}, \hat{\mathscr{E}}\right)=\hat{\mathscr{E}}$ and we may write

$$
h_{m}^{r}=g_{n}^{r}+g_{T}^{1}-\left(\tilde{\mathscr{D}}_{1}+\hat{\mathscr{E}}\right)-\left(\tilde{\mathscr{D}}_{2}-\hat{\mathscr{E}}\right) .
$$

Note that (i), (ii), and (iii) are true for the divisors $\mathscr{D}_{1}=\tilde{\mathscr{D}}_{1}+\hat{\mathscr{E}}$ and $\mathscr{D}_{2}=$ $\mathscr{D}_{2}-\hat{\mathscr{E}}$.

(iv) We may write $h_{m}^{r}=g_{n}^{r}+g_{T}^{1}-\mathscr{D}_{1}-\mathscr{D}_{2}$ where (i), (ii), and (iii) hold for $\mathscr{D}_{1}$ and $\mathscr{D}_{2}$. Let $g_{1}^{r+1}=g_{1}^{r-1}+g_{T}^{1}$ where $g_{n}^{r}=g_{1}^{r-1}+\mathscr{D}_{1}$. By the argument contained in (iii), $g_{1}^{r+1}$ has dimension $r+1$. We have the relationships $g_{n}^{r}=$ $g_{1}^{r+1}-\overline{\mathscr{D}_{1}}$ and $h_{m}^{r}=g_{1}^{r+1}-\mathscr{D}_{2}$. Since $g_{n}^{r}$ and $h_{m}^{r}$ are distinct, $\left(\mathscr{D}_{2}, \overline{\mathscr{D}_{1}}\right)=$ $\varnothing$.

Remark 1.2.4. In any case where the relationship between two linear series is nongeneric, we shall always assume that the pair of divisors involved enjoy the properties of $\mathscr{D}_{1}$ and $\mathscr{D}_{2}$ described in the preceding lemma. Our next result considers the consequences of $X_{p}$ admitting three or more linear series which, taken pairwise, satisfy the hypotheses of 1.2.1.

Theorem 1.2.5. Suppose that $X_{p}$ possesses three linear series $g_{i}, i=1,2,3$, of the same dimension and degree. Let the dimension of each linear series be $r$. Suppose that the linear series taken pairwise, satisfy the hypotheses of 1.2.1 and the hypothesis that $\sum g_{i}$ is of minimal dimension. Let $g_{T}^{1}$ be the resulting pencil imposing two conditions on each of the $g_{i}$. Let us further assume that $g_{i}-g_{T}^{1}$ is simple for each $i$ (cf. 1.1.19). We can write $g_{2}=g_{1}+g_{T}^{1}-\mathscr{D}_{1}-\mathscr{D}_{2}$, and $g_{3}=g_{1}+g_{T}^{1}-\mathscr{E}_{1}-\mathscr{E}_{2}$ for some $\mathscr{D}_{1}, \mathscr{D}_{2}, \mathscr{E}_{1}$, and $\mathscr{E}_{2}$. Then $\left\{\mathscr{D}_{1}, \mathscr{D}_{2}\right\}$ $\cap\left\{\mathscr{E}_{1}, \mathscr{E}_{2}\right\} \neq \varnothing$.

Proof. We have the two relationships

$$
g_{2}=g_{1}+g_{T}^{1}-\mathscr{D}_{1}-\mathscr{D}_{2}
$$

and

$$
g_{3}=g_{1}+g_{T}^{1}-\mathscr{E}_{1}-\mathscr{E}_{2}
$$

By the same type of argument used to produce $(D)$ and $(E)$, we can obtain

$$
g_{3}=g_{2}+g_{T}^{1}-\mathscr{F}_{1}-\mathscr{F}_{2} .
$$

At this stage, there are eight possibilities depending upon whether or not (D), $(E)$, and $(F)$ are generic or nongeneric. By symmetry, we are left with the six cases:

(i) (D), (E), and (F) all generic. 
(ii) (D), (E), and (F) nongeneric.

(iii) (D) and (F) generic, (E) nongeneric.

(iv) (D) and (E) generic, (F) nongeneric.

(v) (D) generic, (E) and (F) nongeneric.

(vi) (D) and (E) nongeneric, (F) generic.

(i) From the three relationships, we find that

$$
g_{3}=g_{1}+2 g_{T}^{1}-\mathscr{D}_{1}-\mathscr{D}_{2}-\mathscr{F}_{1}-\mathscr{F}_{2}
$$

which implies that

$$
g_{3}=\left[g_{1}-g_{T}^{1}\right]+\left[3 g_{T}^{1}-\mathscr{D}_{1}-\mathscr{D}_{2}-\mathscr{F}_{1}-\mathscr{F}_{2}\right] .
$$

However, we also have

$$
g_{3}=\left[g_{1}-g_{T}^{1}\right]+\left[2 g_{T}^{1}-\mathscr{E}_{1}-\mathscr{E}_{2}\right] .
$$

Therefore

$$
2 g_{T}^{1}-\mathscr{E}_{1}-\mathscr{E}_{2} \equiv 3 g_{T}^{1}-\mathscr{D}_{1}-\mathscr{D}_{2}-\mathscr{F}_{1}-\mathscr{F}_{2}
$$

We may rewrite the last relationship as

$$
\overline{\mathscr{E}_{1}}+\overline{\mathscr{E}}_{2} \equiv 3 g_{T}^{1}-\mathscr{D}_{1}-\mathscr{D}_{2}-\mathscr{F}_{1}-\mathscr{F}_{2}
$$

(a) Let us assume that $\mathscr{D}_{1}, \mathscr{F}_{1}$, and $\mathscr{F}_{2}$ each lie in a different divisor of $g_{T}^{1}$. Then we can write

$$
\overline{\mathscr{E}_{1}}+\overline{\mathscr{E}}_{2} \equiv \overline{\mathscr{D}_{1}}+\overline{\mathscr{F}_{1}}+\left[g_{T}^{1}-\mathscr{D}_{2}-\mathscr{F}_{2}\right]
$$

Adding $\mathscr{D}_{2}$ to both sides we obtain

$$
\overline{\mathscr{E}}_{1}+\overline{\mathscr{E}}_{2}+\mathscr{D}_{2}=\overline{\mathscr{D}_{1}}+\overline{\mathscr{F}_{1}}+\overline{\mathscr{F}_{2}}
$$

We have equality here instead of linear equivalence because, by $1.1 .17,3 g_{T}^{1}$ has dimension three and because $\mathscr{D}_{1}, \mathscr{F}_{1}$, and $\mathscr{F}_{2}$ lie in different divisors of $g_{T}^{1}$. We must have, therefore, $\overline{\mathscr{E}_{1}}=\overline{\mathscr{D}_{1}}$, or $\overline{\mathscr{E}}_{2}=\overline{\mathscr{D}_{1}}$, in either case we are done.

(b) Suppose that no three of the divisors $\mathscr{D}_{1}, \mathscr{D}_{2}, \mathscr{F}_{1}, \mathscr{F}_{2}$ are contained in different divisors of $g_{T}^{1}$. Let us assume, for argument's sake, that $\mathscr{F}_{1}$ and $\mathscr{D}_{1}$ are contained in the same divisor of $g_{T}^{1}$, as are $\mathscr{D}_{2}$ and $\mathscr{F}_{2}$. If $\mathscr{D}_{1}+\mathscr{F}_{1}=g_{T}^{1}$, then from (1.4), we obtain

$$
\overline{\mathscr{E}}_{1}+\overline{\mathscr{E}}_{2} \equiv\left[g_{T}^{1}-\mathscr{D}_{1}-\mathscr{F}_{1}\right]+\overline{\mathscr{F}_{2}}+\overline{\mathscr{D}}_{2}
$$

This implies that

$$
\overline{\mathscr{E}_{1}}+\overline{\mathscr{E}}_{2}=\overline{\mathscr{D}}_{2}+\overline{\mathscr{F}_{2}} \text {. }
$$

We have equality here, not just linear equivalence, because $\mathscr{E}_{1}$ and $\mathscr{E}_{2}$ lie in different divisors of $g_{T}^{1}$. However, this relationship implies that $\mathscr{D}_{2}$ and $\mathscr{F}_{2}$ are contained in different divisors of $g_{T}^{1}$, which contradicts our assumption.

If $\left(\overline{\mathscr{D}}_{1}, \mathscr{F}_{1}\right) \neq \varnothing$, then since $\overline{\mathscr{D}_{1}}$ and $\mathscr{F}_{1}$ both impose one condition upon $g_{2}$, we conclude that $\overline{\mathscr{D}_{1}}=\mathscr{F}_{1}$, i.e., $\mathscr{D}_{1}+\mathscr{F}_{1}=g_{T}^{1}$. However, we have already disposed of this possibility.

Finally, we must consider the case $\left(\overline{\mathscr{D}}_{1}, \mathscr{F}_{1}\right)=\varnothing$. Then, since $\mathscr{D}_{1}$ and $\mathscr{F}_{1}$ are contained in the same divisor of $g_{T}^{1},\left(\mathscr{D}_{1}, \mathscr{F}_{1}\right)=\mathscr{F}_{1}$. Similarly, $\left(\mathscr{F}_{2}, \mathscr{D}_{2}\right)=$ 
$\mathscr{F}_{2}$. Suppose that there exist divisors $\mathscr{D}$ and $\mathscr{E}$ such that $\mathscr{F}_{1}+\mathscr{D}=\mathscr{D}_{1}$ and $\mathscr{F}_{2}+\mathscr{E}=\mathscr{D}_{2}$. Then

$$
\begin{aligned}
& g_{2}=g_{1}+g_{T}^{1}-\mathscr{D}_{1}-\mathscr{D}_{2}, \\
& g_{3}=g_{1}+g_{T}^{1}-\mathscr{C}_{1}-\mathscr{C}_{2}, \\
& g_{3}=g_{2}+g_{T}^{1}-\mathscr{D}_{1}-\mathscr{D}_{2}+\mathscr{D}+\mathscr{E} .
\end{aligned}
$$

Since $g_{2}$ and $g_{3}$ have the same degree, we must have $\mathscr{D}=\mathscr{E}=0$. If this is the case, and we add $g_{1}$ to the second expression for $g_{3}$, then we find that $g_{1}+g_{3}=2 g_{2}$, counting dimensions leads us to a contradiction.

(ii) and (vi) (a) First of all, let us assume that $\mathscr{D}_{i}$ and $\mathscr{E}_{i}$ lie in different divisors of $g_{T}^{1}$. Suppose that $\mathscr{D}_{1}$ lies in $\mathscr{H}$, and $\mathscr{E}_{1}$ lies in $\mathscr{G}$, then $(\mathscr{H}, \mathscr{G})=$ $\varnothing$. We shall obtain a contradiction as follows. We have the relationship

$$
\overline{\mathscr{E}}_{1}+\overline{\mathscr{E}}_{2} \equiv 3 g_{T}^{1}-\mathscr{D}_{1}-\mathscr{D}_{2}-\mathscr{F}_{1}-\mathscr{F}_{2} \text {. }
$$

The left-hand side has dimension zero, since $\mathscr{E}_{1}+\mathscr{E}_{2}$ is not contained in a single divisor of $g_{T}^{1}$. Futhermore, it contains only points which lie in $\mathscr{G}$, so adding the divisor $g_{T}^{1}-\overline{\mathscr{D}}_{2}=\mathscr{D}_{2}$ to it does not change the dimension since the result may be written as $3 g_{T}^{1}-\mathscr{E}_{1}-\mathscr{E}_{2}-\overline{\mathscr{D}_{2}}$, which clearly has dimension zero. We have

$$
\mathscr{D}_{2}+\overline{\mathscr{E}}_{1}+\overline{\mathscr{E}}_{2}=\overline{\mathscr{D}_{1}}+\overline{\mathscr{F}_{1}}+\overline{\mathscr{F}_{2}}
$$

Since $\left(\overline{\mathscr{D}_{1}}, \mathscr{D}_{2}\right)=\varnothing,\left(\overline{\mathscr{E}_{1}}+\overline{\mathscr{E}_{2}}, \overline{\mathscr{D}_{1}}\right) \neq \varnothing$, which is a contradiction. This argument is typical of the ones used below in (iii), (iv), and (v), and we shall not reproduce it.

(b) Now we may assume that $\mathscr{D}_{1}$ and $\mathscr{E}_{1}$ lie in the same divisor of $g_{T}^{1}$. We recall that $T=\operatorname{deg} \mathscr{D}_{1}+\operatorname{deg} \mathscr{D}_{2}$ and that $\left(\mathscr{D}_{1}, \mathscr{D}_{2}\right)=\mathscr{D}_{2}$. Therefore, $\operatorname{deg} \mathscr{D}_{1} \geq T / 2$. Exactly the same is true for the pair of divisors $\mathscr{E}_{1}$ and $\mathscr{E}_{2}$.

Suppose that $\operatorname{deg} \mathscr{D}_{1}>T / 2$. We conclude that $\left(\mathscr{D}_{1}, \mathscr{C}_{1}\right) \neq \varnothing$. Since $g_{1}-\mathscr{D}_{1}$ and $g_{1}-\mathscr{E}_{1}$ are both fixed point free, and both have dimension $r-1, \mathscr{D}_{1}=\mathscr{E}_{1}$.

If $\operatorname{deg} \mathscr{D}_{1}=T / 2$, then $\mathscr{D}_{2}=\mathscr{D}_{1}$. If $\left(\mathscr{D}_{1}, \mathscr{C}_{1}\right)=\varnothing$, then the restriction on the degrees demands that $\mathscr{E}_{1}=\mathscr{E}_{2}=\overline{\mathscr{D}_{1}}$. Adding $g_{2}$ and $g_{3}$ together, we find that $g_{2}+g_{3}=2 g_{1}$. Counting dimensions gives a contradiction, so $\left(\mathscr{D}_{1}, \mathscr{E}_{1}\right) \neq \varnothing$, in which case, we must have $\mathscr{D}_{1}=\mathscr{E}_{1}$.

(iii) We have $\left(\mathscr{E}_{1}, \mathscr{E}_{2}\right)=\mathscr{E}_{2}$. Suppose that $\mathscr{E}_{1}$ is contained in $\mathscr{H}$, a divisor of $g_{T}^{1}$. Since $\mathscr{D}_{1}$ and $\mathscr{D}_{2}$ are in different divisors of $g_{T}^{1}$, they both cannot lie inside $\mathscr{H}$.

(a) Suppose that neither $\mathscr{D}_{1}$ nor $\mathscr{D}_{2}$ lies in $\mathscr{H}$. Then, as in (i) we find that since $\overline{\mathscr{E}}_{1}+\overline{\mathscr{E}}_{2}$ has dimension zero

$$
\mathscr{D}_{2}+\overline{\mathscr{E}}_{1}+\overline{\mathscr{E}}_{2}=\overline{\mathscr{D}_{1}}+\overline{\mathscr{F}_{1}}+\overline{\mathscr{F}_{2}}
$$

Since $\mathscr{F}_{1}$ and $\mathscr{F}_{2}$ are contained in different divisors of $g_{T}^{1}$, we must have, for example, $\mathscr{D}_{2}=\overline{\mathscr{F}_{1}}$, and therefore $\overline{\mathscr{E}}_{1}+\overline{\mathscr{E}}_{2}=\overline{\mathscr{D}_{1}}+\overline{\mathscr{F}}$ which contradicts the fact that $\left(\mathscr{D}_{1}, \mathscr{H}\right)=\varnothing$.

(b) Suppose that $\mathscr{D}_{1}$ lies in $\mathscr{H}$, then $\mathscr{D}_{2}$ certaintly lies in a different divisor and, once again, we have

$$
\mathscr{D}_{2}+\overline{\mathscr{E}}_{1}+\overline{\mathscr{E}}_{2}=\overline{\mathscr{D}_{1}}+\overline{\mathscr{F}_{1}}+\overline{\mathscr{F}_{2}} .
$$

As before, we can reduce this relationship to

$$
\overline{\mathscr{E}}_{1}+\overline{\mathscr{E}}_{2}=\overline{\mathscr{D}_{1}}+\overline{\mathscr{F}}_{2}
$$


We note that since $\left(\overline{\mathscr{E}}_{2}, \overline{\mathscr{E}}_{1}\right)=\overline{\mathscr{E}}_{1},\left(\overline{\mathscr{E}}_{2}, \overline{\mathscr{F}}_{2}\right) \neq \varnothing$. However, we have the two relationships

$$
g_{3}=g_{1}+g_{T}^{1}-\mathscr{E}_{1}-\mathscr{E}_{2} \quad \text { and } \quad g_{3}=g_{2}+g_{T}^{1}-\mathscr{F}_{1}-\mathscr{F}_{2} .
$$

From 1.2 .2 and 1.2 .3 , it follows that $\overline{\mathscr{E}}_{2}$ and $\overline{\mathscr{F}_{2}}$ both impose one condition on $g_{3}$. Since both $g_{3}-\overline{\mathscr{E}}_{2}$ and $g_{3}-\overline{\mathscr{F}}_{2}$ are fixed point free, we conclude that $\overline{\mathscr{E}_{2}}=\overline{\mathscr{F}}$ and that $\overline{\mathscr{E}_{1}}=\overline{\mathscr{D}_{1}}$, i.e., $\mathscr{E}_{1}=\mathscr{D}_{1}$.

(iv) In this case, we may assume that $\mathscr{D}_{1}$ is not contained in the same divisor of $g_{T}^{1}$ as $\mathscr{F}_{1}$ is. Again, we can write

$$
\mathscr{D}_{2}+\overline{\mathscr{E}}_{1}+\overline{\mathscr{E}}_{2}=\overline{\mathscr{D}_{1}}+\overline{\mathscr{F}_{1}}+\overline{\mathscr{F}_{2}} \text {. }
$$

Since $\mathscr{D}_{2}$ and $\mathscr{D}_{1}$ lie in different divisors, and $\mathscr{E}_{1}$ and $\mathscr{E}_{2}$ also lie in different divisors of $g_{T}^{1}$, we must have $\overline{\mathscr{D}_{1}}=\overline{\mathscr{E}_{1}}$ or $\overline{\mathscr{D}_{1}}=\overline{\mathscr{E}_{2}}$.

(v) We shall see that this case is impossible.

(a) Suppose that neither $\mathscr{D}_{1}$ nor $\mathscr{D}_{2}$ is contained in the same divisor of $g_{T}^{1}$ as $\mathscr{E}_{1}$ and $\mathscr{E}_{2}$ are. Then, as before, the linear series $\mathscr{D}_{2}+\overline{\mathscr{E}}_{1}+\overline{\mathscr{E}}_{2}$ will have dimension zero, and we find that

$$
\mathscr{D}_{2}+\overline{\mathscr{E}}_{1}+\overline{\mathscr{E}}_{2}=\overline{\mathscr{D}_{1}}+\overline{\mathscr{F}_{1}}+\overline{\mathscr{F}_{2}} \text {. }
$$

Since $\mathscr{D}_{1}$ and $\mathscr{D}_{2}$ lie in different divisors of $g_{T}^{1}$, we must have $\left(\overline{\mathscr{E}_{1}}+\overline{\mathscr{E}_{2}}, \overline{\mathscr{D}_{1}}\right) \neq$ $\varnothing$, which is a contradiction.

(b) Next, suppose that $\mathscr{D}_{1}$ lies in the same divisor of $g_{T}^{1}$ as $\mathscr{E}_{1}$ and $\mathscr{E}_{2}$ do. Once again, the divisor $\mathscr{D}_{2}+\overline{\mathscr{E}}_{1}+\overline{\mathscr{E}}_{2}$ will have dimension zero and we obtain

$$
\mathscr{D}_{2}+\overline{\mathscr{E}}_{1}+\overline{\mathscr{E}}_{2}=\overline{\mathscr{D}_{1}}+\overline{\mathscr{F}_{1}}+\overline{\mathscr{F}_{2}} \text {. }
$$

At this point, if we assume that $\mathscr{E}_{1}$ and $\mathscr{F}_{1}$ lie in the same divisor of $g_{T}^{1}$, we get a contradiction because $\mathscr{D}_{2}$ and $\mathscr{D}_{1}$ lie in different divisors of $g_{T}^{1}$.

If $\mathscr{E}_{1}$ and $\mathscr{F}_{1}$ lie in different divisors, then we obtain the relationships $\mathscr{D}_{2}=$ $\overline{\mathscr{F}_{1}}+\overline{\mathscr{F}_{2}}$ and $\overline{\mathscr{D}_{1}}=\overline{\mathscr{E}}_{1}+\overline{\mathscr{E}}_{2}$. Considering degrees, $\mathscr{D}_{2}$ must have degree $T$, a contradiction.

Remark 1.2.6. If we have $j \geq 5$ linear series of the same degree and dimension, and a linear combination of these linear series attains equality in the generalised Castelnuovo inequality, then from 1.2.5 and the observation that if four or more sets each contain two elements, and no two sets are disjoint, one element is common to all of the sets, it follows that there exists a divisor $\mathscr{D}$ lying in a single divisor of $g_{T}^{1}$ so that, for $2 \leq i \leq j, g_{i}=g_{1}+g_{T}^{1}-\mathscr{D}-\mathscr{D}_{i}$. Moreover, since $g_{1}-\mathscr{D}$ is base point free (by 1.2 .2 and 1.2.3), $g_{i}-g_{T}^{1}$ is base point free $\forall i$, and

$$
g_{i}-g_{T}^{1}=\left[g_{n}^{r}-\mathscr{D}\right]-\mathscr{D}_{i}
$$

it follows that $\left(\mathscr{D}_{i}, \mathscr{D}_{k}\right)=\varnothing$ if $i \neq k$.

However, if $j=4$ and the relationships between the linear series are generic then there may exist three distinct divisors $\mathscr{D}, \mathscr{E}$, and $\mathscr{F}$, all having the same degree and all lying in different divisors of $g_{T}^{1}$ so that

$$
g_{2}=g_{1}+g_{T}^{1}-\mathscr{D}-\mathscr{E}, \quad g_{3}=g_{1}+g_{T}^{1}-\mathscr{D}-\mathscr{F},
$$

and

$$
g_{4}=g_{1}+g_{T}^{1}-\mathscr{E}-\mathscr{F} .
$$


We shall describe this situation as exceptional.

Remark 1.2.7. If $X_{p}$ admits two linear series $g_{1}$ and $g_{2}$, satisfying the conditions of 1.2.1, then for some divisors $\mathscr{D}_{1}$ and $\mathscr{D}_{2}, g_{2}=g_{1}+g_{T}^{1}-\mathscr{D}_{1}-\mathscr{D}_{2}$. If $\left(\mathscr{D}_{1}, \mathscr{D}_{2}\right)=\varnothing$, then when we map $X_{p}$ to its image in $\mathbf{P}^{r}$ via $g_{1}, \mathscr{D}_{1}$ and $\mathscr{D}_{2}$ will yield singularities which may fall together on the curve in $\mathbf{P}^{r}$, however, by assuming that the singularities are disjoint, we can give a lower bound on their contribution to the delta invariant. In $\S 3$, we show that for generalised Castelnuovo curves, this estimate is sharp. If $\left(\mathscr{D}_{1}, \mathscr{D}_{2}\right)=\mathscr{D}_{2}$, then the singularities on the image curve are not disjoint, one "lives inside the other." We can see this in some sense by using the following rather heuristic argument. The larger divisor $\mathscr{D}_{1}$ corresponds to a singularity $S$ on the curve in $\mathbf{P}^{r}$, the projection of the curve into $\mathbf{P}^{r-1}$ from the "point" $S$ corresponds to the series $g_{m}^{r-1}=g_{1}-\mathscr{D}_{1}$. Since $g_{2}-\overline{\mathscr{D}_{2}}$ has dimension $r-2$ the divisor $\mathscr{D}_{2}$ corresponds to a singular point on the curve in $\mathbf{P}^{r-1}$. In any case the contribution of these nested singularities to the delta-invariant is bounded below by the same lower-bound as in the case $\left(\mathscr{D}_{1}, \mathscr{D}_{2}\right)=\varnothing$. Therefore, as far as the calculations in $\S 3$ are concerned, we may always assume that the relationship between any two linear series is generic.

In this section, we break some ground in our classification of generalised Castelnuovo curves. We perform the preliminary calculations that we will call upon in $\S 3$ when we get down to the classification proper. We can use these results to narrow our range of possible candidates, but alone they are not quite enough to provide models of such curves. Allied to a little geometry, however, they will allow us to complete the classification.

2.0.

Remark 2.0.1. Given positive integers $n, r$, and $j$, consider $n \bmod (j r-1)$. There exists an $l \in \mathbf{Z}^{+}$, so that if $\Lambda=n-(j r-1) l$, then $2-(j-2) r \leq \Lambda \leq 2 r$. We choose $k, 0 \leq k \leq j-1$, so that

$$
\begin{array}{cc}
r+1 \leq \Lambda \leq 2 r & \text { if } k=0 \\
2 \leq \Lambda \leq r & \text { if } k=1 \\
\vdots & \\
2-(k-1) r \leq \Lambda \leq 1-(k-2) r & \text { if } 2 \leq k \leq j-1 .
\end{array}
$$

If $k \leq 1$ we can write $n=(j l-k+2) r-(l+t)$. If $k=0$ then $0 \leq t \leq r-1$, while if $k=1$ then $0 \leq t \leq r-2$. If $k \geq 2$, we write $n=(j l-k+2) r-(l-1+t)$ where $0 \leq t \leq r-1$.

Let us assume that we have a Riemann surface of genus $p, X_{p}$, which admits $j$ simple linear series of dimension $r$ and degree $n$ and that $X_{p}$ is a generalised Castelnuovo curve, by the preceding remark, given $n, r$, and $j$ there are essentially three possiblilities:

(i) $n=(j l+2) r-(l+t)$ for some $t, 0 \leq t \leq r-1 \quad(k=0)$.

(ii) $n=(j l+1) r-(l+t)$ for some $t, 0 \leq t \leq r-2(k=1)$.

(iii) $n=(j l-k+2) r-(l+t-1)$ for some $k, 2 \leq k \leq j-1$ and $t$, $0 \leq t \leq r-1$. 
Perhaps some motivation for the preceding decomposition of $n$, the degree of our linear series, is in order. Let $X_{p}$ be a generalised Castelnuovo curve admitting $j$ linear series of degree $n$ and dimension $r$, and let $k, l$, and $t$ be as above then

(i) If $k=0$, and the linear series

$$
W(j, 0, l)=l\left[g_{1}+g_{2}+\cdots+g_{j}\right]
$$

has smallest possible dimension, then its Clifford index is nonnegative, while $W(j, j-1, l+1)=W(j, 0, l)+g_{1}$ will have negative Clifford index and be nonspecial. ${ }^{1}$

(ii) If $k \geq 1$, and

$$
W(j, k, l)=(l-1)\left[g_{1}+\cdots+g_{k}\right]+l\left[g_{k+1}+\cdots+g_{j}\right]
$$

is of smallest dimension then its Clifford index will be nonnegative. while $W(j, k-1, l)=W(j, k, l)+g_{1}$ will have negative Clifford index and be nonspecial.

In the following calculations, we find the conditions on $n$ that $W(j, j-1, l+1)$ and $W(j, k, l)$ have negative Clifford index. We must also consider the possibility that either $W(j, j-1, l+1)$ or $W(j, k, l)$ is exactly canonical, and has smallest dimension.

We recall that if $g_{1} \cdots g_{j}$ are $j \quad r$-dimensional linear series, then Accola's formula for the minimum dimension of $l_{1} g_{1}+\cdots+l_{j} g_{j}$, is given by

$$
\Psi(j, k, l)=\sum_{i=1}^{j} \frac{\left(l_{i}+1\right)\left(l_{i}\right)}{2} r-\frac{\left(l_{i}\right)\left(l_{i}-1\right)}{2}+\sum_{k=1}^{j} \sum_{i<k} l_{i} l_{k} r .
$$

In [1], Accola derives $\Psi(j, k, l)$ and writes it in a form that enables one to easily see that it is indeed a lower bound on the dimension of $W(j, k, l)$. In the following lemma, we express Accola's estimate for $\Psi(j, k, l)$ in a form that allows us to quickly determine whether or not $W(j, k, l)$ is nonspecial.

Lemma 2.0.2. Let $X_{p}$ be Riemann surface admitting $j$ linear series of dimension $r$ so that

$$
W(j, k, l)=(l-1)\left[g_{1}+\cdots+g_{k}\right]+l\left[g_{k+1}+\cdots+g_{j}\right] 1 \leq k \leq j-1
$$

or

$$
W(j, 0, l)=l\left[g_{1}+\cdots+g_{j}\right]
$$

has the smallest possible dimension $\Psi(j, k, l)$. Then

Proof. Let

$$
\Psi(j, k, l)=\frac{(j l-k+1)(j l-k)}{2} r-\frac{(j l-2 k)(l-1)}{2} .
$$

$$
\Phi(j, k, l)=\frac{(j l-k+1)(j l-k)}{2} r-\frac{(j l-2 k)(l-1)}{2} .
$$

Fixing $j$ and $l$, we proceed, by induction on $k$, to show that $\Phi(j, k, l)=$ $\Psi(j, k, l)$. For $k=j-1$ this assertion is easily verifiable and we omit the details. Let $\theta_{k}$ be the number of conditions that $g_{k}$ imposes on

\footnotetext{
${ }^{1}$ Here, for our convenience we interchange the roles of $g_{1}$ and $g_{j}$.
} 
$W(j, k-1, l)$. From Accola's definition of $\Psi(j, k, l)$ it follows automatically that $\Psi(j, k-1, l)-\theta_{k}=\Psi(j, k, l)$. We note that $\theta_{k}=(j l-k+1) r-(l-1)$ and leave the reader to verify that $\Phi(j, k-1, l)-\theta_{k}=\Phi(j, k, l)$, which completes the proof.

Remark 2.0.3. Usually it is more convenient to write

$$
\Psi(j, k, l)=\frac{(j l-k+1)(j l-k)}{2} r-\frac{(j l-k)(l-1)}{2}+\frac{k(l-1)}{2} .
$$

If $k \geq 1$ and the Clifford index of $W(j, k-1, l)$ is negative, then

$$
n<(j l-k+2) r-(l-1)+\left[\frac{(k-1)(l-1)}{j l-k+1}\right],
$$

and $W(j, k-1, l)$ is nonspecial by Clifford's theorem. We now indicate how the decomposition of $n$ mentioned earlier comes about. We exhibit the case $k=0$ and leave the verification for the cases $k=1$ and $2 \leq k \leq j-1$ to the reader.

(i) $(k=0)$ Using Accola's formula to calculate the dimension of $W(j, j-1, l+1)$, we find it to be (for convenience we interchange the role of $g_{1}$ and $\left.g_{j}\right)$

$$
\Psi(j, j-1, l+1)=\frac{(j l+1)(j l+2)}{2} r-\frac{(j l+1) l}{2}+\frac{(j-1)(l)}{2} .
$$

The degree of $W(j, j-1, l+1)$ is $(j l+1) n$. Thus requiring $W(j, j-1, l+1)$ to have negative Clifford index, and so be nonspecial, means that

$$
(j l+1) n<(j l+1)(j l+2) r-(j l+1)(l)+(j-1) l .
$$

Therefore,

$$
n<(j l+2) r-l+\left[\frac{(j-1) l}{j l+1}\right] .
$$

The quantity in the brackets is always positive and less than 1 , therefore

$$
n \leq(j l+2) r-l \text {. }
$$

At the same time however, we must also have

$$
n \geq(l+1)(r-1)+(j-1) l r+1=(j l+1) r-l
$$

in order to ensure that Castelnuovo's method may be applied. Taking $n=$ $(j l+2) r-(l+t)$ where $0 \leq t \leq r-1$ ensures that both of these conditions are fulfilled.

Remark 2.0.4. We note that $(j l-k) n \neq 2 \Psi(j, k, l)$ unless we take $k=0$ and have $n=(j l+1) r-(l-1)$. However, this is just case (i) with $t=r-1$. We note that, generally speaking, if $W(j, k, l)$ has negative Clifford index then we cannot apply Accola's method to compute the dimension of $W(j, k-1, l)$, the obstruction being the restriction on the degree. However, by the preceding observation, if $W(j, 0, l)$ is exactly canonical and has smallest possible dimension, then $W(j, j-1, l+1)$ is nonspecial and has smallest possible dimension.

Remark 2.0.5. Note that in the case $k \geq 1$ mentioned above we neccessarily have $l \geq 2$. This implies that any pair of $g_{i}$, for $1 \leq i \leq j$, satisfy the 
hypotheses of 1.2.1 namely that $2 g_{i}+2 g_{k}$ has dimension $10 r-2$. This is not the case if $k=0$ and $\sum g_{i}$ is exactly canonical therefore whenever we consider the case $k=0$ we assume that $l \geq 2$.

Finally, the following lemma will be used repeatedly in the following sections. From our work in $\S 1$ we know that a generalised Castelnuovo curve will possess a unique $g_{T}^{1}$ imposing two conditions on each $g_{n}^{r}$ admitted by $X_{p}$. Our next result, due to Accola in [1], gives us a lower bound on the value of $T$. We present the result in its full generality.

Lemma 2.0.6. If $g_{T}^{1}$ is a complete linear series imposing two conditions on each $g_{n_{i}}^{r_{i}}$ for $1 \leq i \leq j$ where each $g_{n_{i}}^{r_{i}}$ is simple and

$$
l_{1} g_{n_{1}}^{r_{1}}+\cdots+l_{j} g_{n_{j}}^{r_{j}}+g_{m}^{\mu}=\mathbf{K}
$$

then $T \geq \sum_{i=1}^{j} l_{i}+2$. If $g_{m}^{\mu}$ is simple, then $T \geq \sum_{i=1}^{j} l_{i}+3$.

Proof. The argument presented here is culled directly from Accola [1]. Assume that $g_{m}^{\mu}$ is composite, for our purposes we shall also assume that it is compounded of $g_{T}^{1}$. Suppose now that $T \leq \sum_{i=1}^{j} l_{i}+1$. Choose a divisor $\mathscr{D}$ in $g_{T}^{1}$, away from the fixed points of $g_{m}^{\mu}$, consisting of $T$ distinct points. Write $\mathscr{D}=x_{1}+\cdots+x_{T}$. Since all of the $g_{n_{i}}^{r_{i}}$ are simple and $g_{T}^{1}$ imposes two conditions on each of them we may choose $T-1$ divisors (indexed in some fashion) $\mathscr{D}_{i} \in g_{n_{k}}^{r_{k}}$ for some $k \in\{1, \ldots, j\}$ so that $\left(\mathscr{D}, \mathscr{D}_{i}\right)=x_{i}$ (such divisors exist by Accola's general position theorem). Now consider the divisor $\mathscr{Q}=\sum \mathscr{D}_{i}$. It lies in $\mathbf{K}$ and contains $T-1$ points of $\mathscr{D}$. Since $g_{T}^{1}$ imposes $T-1$ conditions on $\mathbf{K}$ we see that $\mathscr{Q}$ contains all of the points of $\mathscr{D}$, which is a contradiction. When $g_{m}^{\mu}$ is simple, then $g_{T}^{1}$ imposes at least two conditions on it, and the argument is essentially the same except that in addition to the $\mathscr{D}_{i}$ mentioned above we can find a divisor $\mathscr{D}_{0}$ in $g_{m}^{\mu}$ so that $\left(\mathscr{D}_{0}, \mathscr{D}\right)=x_{i}$, for some $i$, then we let $Q=\mathscr{D}_{0}+\sum \mathscr{D}_{i}$.

Remark 2.0.7. If $X_{p}$ is a generalised Castelnuovo curve admitting $j$ linear series $g_{1}, \ldots, g_{j}$ all of dimension $r$ and degree $n$ so that $W(j, k+1, l)$ is nonspecial while $W(j, k, l)$ is special then, denoting the canonical series of $X_{p}$ by $\mathbf{K}$, the linear series

$$
g_{m}^{\mu}=\mathbf{K}-W(j, k, l)
$$

will be called the residual series. It may be simple or composite, and be base point free or have base points. In the following section we shall assume that if the residual series is simple then it is also base point free, we shall justify this assumption in $\S 3$.

2.1. The case $k=0, l \geq 2$. Since $W(j, j-1, l+1)=W_{0}$ is nonspecial, we proceed to use its degree and dimension to calculate the genus of our curve. We find that

$$
p=\frac{(j l+1)(j l+2)}{2} r-\frac{j l(l+1)}{2}-(j l+1) t .
$$

Since $W_{1}=W_{0}-g_{1}$ is special, for suitable $m$ and $\mu$, we have

$$
l\left[g_{1}+\cdots+g_{j}\right]+g_{m}^{\mu}=\mathbf{K} .
$$

A direct calculation shows that $m=(j l+2)(r-t-1)$. Calculating $\mu$ is a little more subtle, we assign to the degree and dimension of $W_{0}$ the variables 
$\Delta_{0}$ and $\boldsymbol{\theta}_{0}$, respectively. Next, we assign to the degree and dimension of $W_{1}$ the variables $\Delta_{1}$ and $\theta_{1}$, respectively. We claim that

$$
\mu=\left[\Delta_{0}-\Delta_{1}\right]-\left[\Theta_{0}-\Theta_{1}\right]-1 \text {. }
$$

From the Brill-Nöether form of the Riemann-Roch Theorem, $m-2 \mu=\Delta_{1}-$ $2 \boldsymbol{\theta}_{1}$. Since $m=2 p-2-\Delta_{1}$ and $p=\Delta_{0}-\boldsymbol{\theta}_{0}$, our claim follows. Clearly, $\Delta_{0}-\Delta_{1}=n$, so it remains to compute the value of $\Theta_{0}-\Theta_{1}$. However, this quantity is exactly the number of conditions that $g_{1}$ imposes on $W_{0}$. Since everything is "tight," 2 it is equal to $(j-1) l r+(l+1)(r-1)+1=j l r+r-l$. Therefore, since $n=(j l+2) r-(l+t)$,

$$
\mu=r-1-t \text {. }
$$

We consider the linear series $L_{1}=g_{1}+g_{m}^{r-t-1}$ and $L_{2}=W_{1}-g_{1}$. Since $L_{1}+L_{2}=\mathbf{K}$, Brill-Nöether implies that both series have the same Clifford index, and we can use this fact to calculate the dimension of $L_{1}$. Let this quantity be $\mu_{1} \cdot{ }^{3}$ The degree of $L_{1}$ equals $2 n-(j-1) l-(j l+1) t-2$, whereas the degree of $L_{2}$ is $\Delta_{1}-n$ and its dimension equals $\theta_{1}-\theta_{1}$ where $\theta_{1}$ is the number of conditions that $g_{1}$ imposes on $W_{1}$. We have

$$
2 n-(j-1) l-(j l+1) t-2-2 \mu_{1}=\left[\Delta_{1}-2 \theta_{1}\right]-\left[n-2 \theta_{1}\right] .
$$

By "tightness", $\theta_{1}=(j-1)(l) r+l(r-1)+1$ and we find that

$$
\mu_{1}=3 r-2 t-2 \text {. }
$$

Remark 2.1.1. If $t \geq 1$ then $g_{m}^{\mu}$ is composite, if $t \geq 2$ it follows directly from 1.1.4, while if $t=1$ and $g_{m}^{r-2}$ is simple then 1.1 .4 implies that it imposes $r-2$ conditions on each of the $g_{i}$, this in turn implies the existence of $j$ distinct $g_{n-m}^{1}$ 's each imposing two conditions on $g_{i} \forall i$, this is impossible. If $g_{m}^{\mu}$ is composite then, by 2.0 .6 and 1.1 .5 , it is compounded of a $g_{j l+2}^{1}$ which imposes two conditions on each $g_{i}$.

Remark 2.1.2. If $t=0$, then $g_{m}^{r-1}$ may be simple or composite. If $g_{m}^{r-1}$ is simple, then $X_{p}$ admits a $g_{T}^{1}$ which imposes two conditions on each of the $g_{i}$ and also on $g_{m}^{r-1}$, by $2.0 .6, T \geq j l+3$. If $g_{m}^{r-1}$ is base point free then 1.1 .4 implies the existence of an effective divisor $\mathscr{D}$ so that

$$
g_{1}=g_{m}^{r-1}+\mathscr{D} \text {. }
$$

If $g_{m}^{r-1}$ has base points and if $g_{m-\varepsilon}^{r-1}$ is the moving part of the series we have $g_{1}=g_{m-\varepsilon}^{r-1}+\mathscr{D}$. From 1.1.20 we see that the divisor $\mathscr{D}$, above, is contained in a single divisor of $g_{T}^{1}$.

Lemma 2.1.3. Suppose we are in the situation mentioned in 2.1 .2 then by Theorem 1.2.1 there exist divisors $\mathscr{D}_{1}$ and $\mathscr{D}_{2}$ so that

$$
g_{2}=g_{1}+g_{T}^{1}-\mathscr{D}_{1}-\mathscr{D}_{2} \text {. }
$$

(i) If the relationship between $g_{1}$ and $g_{2}$ is generic, then either $\mathscr{D}_{1}=\mathscr{D}$ or $\mathscr{D}_{2}=\mathscr{D}$.

(ii) If the relationship between the two is nongeneric, i.e., $\left(\mathscr{D}_{1}, \mathscr{D}_{2}\right)=\mathscr{D}_{2}$, then $\mathscr{D}=\mathscr{D}_{1}$.

\footnotetext{
${ }^{2}$ From now on we shall be dealing with the case where all of the previous inequalities in estimates of the dimension are equalities. We shall refer to this as tightness or sharpness.

${ }^{3}$ Obviously $g_{i}+g_{m}^{\mu}$ has dimension $\mu_{1}$ for $i=1, \ldots, j$.
} 
Proof. We may suppose that $g_{m}^{r-1}$ is fixed point free. Substituting for $g_{1}$ in (2.2) we find that

$$
g_{2}=g_{m}^{r-1}+\mathscr{D}+g_{T}^{1}-\mathscr{D}_{1}-\mathscr{D}_{2} .
$$

The dimension of $g_{2}+g_{m}^{r-1}$ is $\mu_{1}=3 r-2$. Therefore, there exists a divisor $\mathscr{E}$ so that $g_{2}=g_{m}^{r-1}+\mathscr{E}$. It follows that

$$
\mathscr{E} \equiv g_{T}^{1}+\mathscr{D}-\mathscr{D}_{1}-\mathscr{D}_{2} .
$$

There are two possibilities to consider:

(i) If we are in the generic situation then $\mathscr{D}, \mathscr{D}_{1}$, and $\mathscr{D}_{2}$ cannot all lie in the same divisor of $g_{T}^{1}$. Let us assume that $\mathscr{D}_{1}$ and $\mathscr{D}$ lie in distinct divisors of $g_{T}^{1}$. Adding $\mathscr{D}_{2}$ to both sides of our equivalence relation gives

$$
\mathscr{E}+\mathscr{D}_{2}=\left[g_{T}^{1}-\mathscr{D}_{1}\right]+\mathscr{D} .
$$

We have equality because the right-hand side has dimension zero. Noting that $\mathscr{D}_{2}$ and $\overline{\mathscr{D}}_{1}$ lie in distinct divisors of $g_{T}^{1}$, we conclude that $\mathscr{D}=\mathscr{D}_{2}$, and $\mathscr{E}=\overline{\mathscr{D}}_{1}$.

(ii) In the nongeneric situation we may suppose that $\left(\mathscr{D}_{1}, \mathscr{D}_{2}\right)=\mathscr{D}_{2}$, and that $g_{1}-\mathscr{D}_{1}$ has dimension $r-1$ and is fixed point free, as is $g_{1}-\mathscr{D}$. Then, since $\mathscr{D}_{1}+\mathscr{D}_{2}$ has dimension zero,

$$
\mathscr{D}_{1}+\mathscr{D}_{2}=g_{T}^{1}-\mathscr{E}+\mathscr{D} .
$$

Since $\left(\mathscr{D}_{1}, \mathscr{D}\right) \neq \varnothing$, we conclude that $\mathscr{D}_{1}=\mathscr{D}$.

2.2. The case $k=1$. Suppose that $n=(j l+1) r-(l+t) . W_{0}=l\left[g_{1}+\cdots+g_{j}\right]$ is nonspecial, so that we may use its degree and dimension to calculate the genus of $X_{p}$, we find that

$$
p=\frac{(j l+1)(j l)}{2} r-\frac{j l(l+1)}{2}-j l t .
$$

We write the canonical series as

$$
(l-1) g_{1}+l\left[g_{2}+\cdots+g_{j}\right]+g_{m}^{\mu}=\mathbf{K} .
$$

Let us denote by $\mu_{1}$ and $\mu_{2}$, respectively, the dimension of $g_{1}+g_{m}^{\mu}$ and $g_{i}+g_{m}^{\mu}$, for $2 \leq i \leq j$. We may establish the following:
(i) $m=(r-t-2)(j l+1)+(j-1) l$.
(ii) $\mu=r-t-2$.
(iii) $\mu_{1}=r+2 \mu$.
(iv) $\mu_{2}=r+2 \mu+1$, for $2 \leq i \leq j$.

Note that $\mu_{1}$ has attained its mimimum possible value and we may apply 1.1.4 and 1.1.5. There are two possibilities to consider if $t=0$, and one otherwise. If $t=0$ we may be able to renumber our linear series so that $g_{m}^{r-2}$ is simple, this is the case we consider in (a) below. If $t=0$ and no such renumbering is possible or, if $t \geq 1$, then $g_{m}^{r-t-2}$ must be composite and we analyse this case in (b) and 2.2.1.

(a) Suppose that $t=0$, and $g_{m}^{r-2}$ is simple. Noting that $n-m=j l+2$, we find that $X_{p}$ possesses a $g_{j l+2}^{1}$ so that

$$
g_{1}=g_{m}^{r-2}+g_{j l+2}^{1}
$$


Furthermore, $g_{j l+2}^{1}$ imposes two conditions on each $g_{i}$.

(b) Suppose that $g_{m}^{r-t-2}$ is composite. By 1.1 .5 it compounded of a $g_{T}^{1}$ imposing two conditions on $g_{1}$ and $g_{m}^{r-t-2}=(r-t-2) g_{T}^{1}+\mathscr{B}$ for some fixed divisor $\mathscr{B}$. By 2.0.6 $T=j l+\varepsilon+1$ where $\varepsilon \geq 0$. We may write

$$
m=(j l+1+\varepsilon)(r-2-t)+(j-1) l-(r-2-t) \varepsilon,
$$

and so $\mathscr{B}$ has degree $(j-1) l-(r-2-t) \varepsilon$.

We know that $g_{2}=g_{1}+g_{j l+\varepsilon+1}^{1}-\mathscr{D}_{1}-\mathscr{D}_{2}$ and that the sum of the degrees of $\mathscr{D}_{1}$ and $\mathscr{D}_{2}$ is $j l+1+\varepsilon$. The following lemma will give us some more information about the degrees of $\mathscr{D}_{1}$ and $\mathscr{D}_{2}$.

Lemma 2.2.1. $\mathscr{B}$, the base locus of $g_{m}^{r-t-2}$, contains either $\mathscr{D}_{1}$ or $\mathscr{D}_{2}$.

Proof. (i) First we remark that $g_{T}^{1}+\mathscr{B}$ has dimension 1. This is immediate if $t<r-2$, otherwise $(r-t-2) g_{T}^{1}+\mathscr{B}$ would have dimension greater than $r-t-2$. If $t=r-2$, and $g_{1}-g_{T}^{1}$ is simple, then consider $g_{1}+\mathscr{B}$, it has dimension $r$, rewriting it as $\left[g_{1}-g_{T}^{1}\right]+\left[g_{T}^{1}+\mathscr{B}\right]$, we see that $g_{T}^{1}+\mathscr{B}$ cannot have dimension greater than one, if it did $g_{1}+\mathscr{B}$ would have dimension $r-2+4+\varepsilon>r$ where $\varepsilon \geq 0$. If $g_{2}-g_{T}^{1}$ is simple we can use a similar argument.

If $g_{1}=(r-1) g_{T}^{1}+\mathscr{P}$, and $g_{2}=(r-1) g_{T}^{1}+\mathscr{Q}$, then we use the fact that $g_{1}+\mathscr{B}$ has dimension $r$. Write $g_{1}+\mathscr{B}=(r-2) g_{T}^{1}+\left[g_{T}^{1}+\mathscr{B}\right]+\mathscr{P}$. If $g_{T}^{1}+\mathscr{B}$ has dimension two or more, then $g_{1}+\mathscr{B}$ will have dimension at least $2+2(r-2)=2 r-2$, which is a contradiction.

(ii) Let us assume that the relationship between $g_{1}$ and $g_{2}$ is generic. From the relationship

$$
(l-1) g_{1}+l\left[g_{2}+g_{3}+\cdots+g_{j}\right]+(r-t-2) g_{T}^{1}+\mathscr{B}_{1}=\mathbf{K},
$$

we obtain

$$
(l-1) g_{2}+l\left[g_{1}+g_{3}+\cdots+g_{j}\right]+(r-t-2) g_{T}^{1}+\mathscr{B}_{2}=\mathbf{K}
$$

by interchanging the roles of $g_{1}$ and $g_{2}$. Equating these two expressions gives

$$
g_{2}+\mathscr{B}_{1}=g_{1}+\mathscr{B}_{2} \text {. }
$$

We use the fact that

$$
g_{2}=g_{1}+g_{T}^{1}-\mathscr{D}_{1}-\mathscr{D}_{2}=\left[g_{1}-g_{T}^{1}\right]+\overline{\mathscr{D}_{1}}+\overline{\mathscr{D}_{2}}
$$

to obtain

$$
\overline{\mathscr{D}_{1}}+\overline{\mathscr{D}_{2}}+\mathscr{B}_{1}=g_{T}^{1}+\mathscr{B}_{2}
$$

$\mathscr{B}_{1}$ cannot contain both $\mathscr{D}_{1}$ and $\mathscr{D}_{2}$, suppose that $\left(\mathscr{D}_{1}, \mathscr{B}_{1}\right)=\varnothing$. Then, writing $g_{T}^{1}=\overline{\mathscr{D}_{2}}+\mathscr{D}_{2}$, we obtain

$$
\overline{\mathscr{D}_{1}}+\mathscr{B}_{1}=\mathscr{D}_{2}+\mathscr{B}_{2}
$$

We have equality because $g_{T}^{1}+\mathscr{B}_{2}$ has dimension one and $\overline{\mathscr{D}}_{2}$ cannot be contained in $\mathscr{B}_{2}$. For if it were, we would have $\mathscr{B}_{2}=\mathscr{F}+\overline{\mathscr{D}}_{2}$ which would imply that

$$
\overline{\mathscr{D}_{1}}+\mathscr{B}_{1}=g_{T}^{1}+\mathscr{F},
$$

i.e.,

$$
\mathscr{B}_{1}=\mathscr{D}_{1}+\mathscr{F}
$$


which contradicts our assumption that $\left(\mathscr{D}_{1}, \mathscr{B}_{1}\right)=\varnothing$. So we have

$$
\overline{\mathscr{D}_{1}}+\mathscr{B}_{1}=\mathscr{D}_{2}+\mathscr{B}_{2}
$$

which implies that $\mathscr{D}_{2} \subset \mathscr{B}_{1}$.

(iii) Assume now that the relationship between $g_{1}$ and $g_{2}$ nongeneric. By assumption $g_{2}=g_{1}+g_{T}^{1}-\mathscr{D}-\mathscr{D}_{2}$ with $\left(\mathscr{D}, \mathscr{D}_{2}\right)=\mathscr{D}_{2}$. We want to show that either $\left(\mathscr{D}, \mathscr{B}_{1}\right)=\mathscr{D}$ or $\left(\mathscr{D}_{2}, \mathscr{B}_{1}\right)=\mathscr{D}_{2}$. The argument is the same as in the generic case, until we arrive at the relationship

$$
\overline{\mathscr{D}}+\overline{\mathscr{D}_{2}}+\mathscr{B}_{1}=g_{T}^{1}+\mathscr{B}_{2}
$$

If $\left(\mathscr{B}_{2}, \overline{\mathscr{D}_{2}}\right)=\overline{\mathscr{D}_{2}}$, then clearly, $\left(\mathscr{B}_{1}, \mathscr{D}\right)=\mathscr{D}$. If $\left(\mathscr{B}_{2}, \overline{\mathscr{D}_{2}}\right) \neq \overline{\mathscr{D}_{2}}$, then

$$
\overline{\mathscr{D}}+\mathscr{B}_{1}=\mathscr{D}_{2}+\mathscr{B}_{2} \text {. }
$$

Since $\left(\overline{\mathscr{D}}, \mathscr{D}_{2}\right)=\varnothing$, we must have $\left(\mathscr{B}_{1}, \mathscr{D}_{2}\right)=\mathscr{D}_{2}$.

Corollary 2.2.2. If we are not dealing with the exceptional case for $j=4$, and there is no reordering of our linear series which allows case (a) to happen, then $g_{i}=g_{1}+g_{T}^{1}-\mathscr{D}-\mathscr{D}_{i}$ for some divisor $\mathscr{D}$. It follows from 2.2.1 and 1.2.6 that either $\mathscr{D} \subseteq \mathscr{B}$ or $\sum_{i=2}^{j} \mathscr{D}_{i} \subseteq \mathscr{B}$.

2.3. The case $k \geq 2$. Suppose that $n=(j l-k+2) r-(l+t-1)$ for some $k$ with $2 \leq k \leq j-1$ and $0 \leq t \leq r-1$. We saw before that

$$
W_{0}=(l-1)\left[g_{2}+\cdots+g_{k}\right]+l\left[g_{1}+g_{k+1}+\cdots+g_{j}\right]
$$

is nonspecial, and that $W_{1}=W_{0}-g_{1}$ is special. The genus $p$ of our curve is

$$
p=\frac{(j l-k+2)(j l-k+1)}{2} r-\frac{(j l)(l-1)}{2}-(j l-k+1) t .
$$

The canonical series may be written

$$
(l-1)\left[g_{1}+\cdots+g_{k}\right]+l\left[g_{k+1}+\cdots+g_{j}\right]+g_{m}^{\mu}=\mathbf{K} .
$$

Let us denote by $\mu_{1}$ and $\mu_{2}$, respectively, the dimension of $g_{1}+g_{m}^{\mu}$ and $g_{i}+g_{m}^{\mu}$, for $k+1 \leq i \leq j$. We may establish the following

(i) $m=(r-t-1)(j l-k+2)+(j-k) l$.

(ii) $\mu=r-t-1$.

(iii) $\mu_{1}=r+2 \mu$.

(iv) $\mu_{2}=r+2 \mu+1$.

Remark 2.3.1. Note that there is nothing to distinguish $g_{i}$ from $g_{1}$ for $1 \leq i \leq k$ as far as the calculation that $g_{1}+g_{m}^{\mu}$ has dimension $r+2 \mu$ is concerned. Note also that $\mu_{1}$ attains its minimum value and so we can apply 1.1.4 and 1.1.5. In the light of (iv), above, we make the following claim which will be of use in the case $t=0$.

Proposition 2.3.2. If $g_{n}^{r}$ and $g_{m}^{r-1}$ are two fixed point free linear series so that $2 g_{n}^{r}$ has dimension $3 r-1$ and $g_{n}^{r}+g_{m}^{r-1}$ has dimension $3 r-1$, then $g_{m}^{r-1} \nsubseteq g_{n}^{r}$. Proof. Suppose that $g_{n}^{r}=g_{m}^{r-1}+\mathscr{E}$ for some zero-dimensional divisor $\mathscr{E}$. Add $g_{n}^{r}$ to both sides. If $g_{n}^{r}+g_{m}^{r-1}$ has dimension $3 r-1$, then $\mathscr{E}$ is a fixed divisor of $2 g_{n}^{r}$. Since $g_{n}^{r}$ is fixed point free, this is impossible. 
Corollary 2.3.3. If $t=0$ in the situation above and $g_{m}^{\mu}$ is simple and fixed point free, then by 2.3.2 $g_{m}^{\mu}=g_{m}^{r-1} \not \subset g_{k+i}$ for $1 \leq i \leq j-k$.

(a) If $t=0$ and $g_{m}^{r-1}$ is simple and fixed point free (we briefly discuss the possibility that $g_{m}^{r-1}$, has fixed points in $\S 3.3$ ) we may apply 1.1.4 to see that there exists an effective divisor $\mathscr{D}$, so that $|\mathscr{D}|$ has dimension zero and

$$
g_{1}=g_{m}^{r-1}+\mathscr{D} \text {. }
$$

By 1.1.20 $\mathscr{D}$ is contained in some divisor of $g_{T}^{1}$ where $g_{T}^{1}$ imposes two conditions on each of the $g_{i}$ 's and on $g_{m}^{r-1}$. As we remarked above, $g_{i}+g_{m}^{r-1}$ has dimension $3 r-2$ for $1 \leq i \leq k$, and therefore there exist effective divisors $\mathscr{E}_{i}$ so that

$$
g_{i}=g_{m}^{r-1}+\mathscr{E}_{i} \quad \text { for } 1 \leq i \leq k .
$$

The following result will eliminate a range of possible models.

Lemma 2.3.4. If $j \geq 5$ and $k \geq 3$ then $g_{m}^{r-1}$ cannot be simple.

Proof. We consider the case $k=3<j-1$ and $j \geq 5$. By the preceding remark, there exists an effective divisor $\mathscr{D}$ so that

$$
g_{1}=g_{m}^{r-1}+\mathscr{D} \text {. }
$$

From 1.2.1

$$
g_{2}=g_{1}+g_{T}^{1}-\mathscr{D}_{2}-\mathscr{Q}_{2} .
$$

Substituting for $g_{1}$ in the last expression, we find that

$$
g_{2}=g_{m}^{r-1}+\mathscr{D}+g_{T}^{1}-\mathscr{D}_{2}-\mathscr{Q}_{2},
$$

since $g_{2}=g_{m}^{r-1}+\mathscr{E}_{2}$ for some effective divisor $\mathscr{E}_{2}$. We deduce, as in 2.1.2, that $\mathscr{D}=\mathscr{D}_{2}$ or $\mathscr{D}=\mathscr{Q}_{2}$. Suppose the former is the case. Arguing in a similar fashion with $g_{3}$, there exists an effective divisor $\mathscr{Q}_{3}$ so that

$$
g_{3}=g_{1}+g_{T}^{1}-\mathscr{D}-\mathscr{Q}_{3} \text {. }
$$

However, we also have

$$
g_{4}=g_{1}+g_{T}^{1}-\mathscr{D}_{4}-\mathscr{Q}_{4} \text { and } g_{5}=g_{1}+g_{T}^{1}-\mathscr{D}_{5}-\mathscr{Q}_{5} .
$$

Consider our expressions for $g_{2}$ and $g_{4}$. We know the divisors $\mathscr{D}_{4}, \mathscr{Q}_{4}, \mathscr{D}, \mathscr{Q}_{2}$ cannot all be distinct. However, we cannot have either $\mathscr{D}_{4}=\mathscr{D}$ or $\mathscr{Q}_{4}=\mathscr{D}$, either of these would imply that $g_{m}^{r-1} \subset g_{4}$, which is false. So we must have $\mathscr{D}_{4}=\mathscr{Q}_{2}$ or $\mathscr{Q}_{4}=\mathscr{Q}_{2}$. For argument's sake let us suppose that the former is the case. By arguing in the same way with $g_{3}$ and $g_{4}$ we find that $\mathscr{Q}_{4}=\mathscr{Q}_{3}$. Therefore

$$
g_{4}=g_{1}+g_{T}^{1}-\mathscr{Q}_{2}-\mathscr{Q}_{3} .
$$

A similar argument for $g_{5}$ gives

$$
g_{5}=g_{1}+g_{T}^{1}-\mathscr{Q}_{2}-\mathscr{Q}_{3} .
$$

Since $g_{4}$ and $g_{5}$ are distinct, we have a contradiction. If $k=j-1$ the argument given above does not go through but $j \geq 5$ implies $k \geq 4$ and so we have $g_{4}=g_{1}+g_{T}^{1}-\mathscr{D}-\mathscr{Q}_{4}$ with $\mathscr{Q}_{4} \notin\left\{\mathscr{Q}_{2}, \mathscr{Q}_{3}\right\}$. However $g_{m}^{r-1} \not \subset g_{j}$ implies that $\mathscr{Q}_{4} \in\left\{\mathscr{Q}_{2}, \mathscr{Q}_{3}\right\}$ which is a contradiction.

(b) If $g_{m}^{r-t-1}$ is composite, there exists a $g_{j l-k+2+\varepsilon}^{1}$ imposing two conditions on $g_{1}$, and $g_{m}^{r-t-1}=(r-t-1) g_{j l-k+\varepsilon+2}^{1}+\mathscr{B}$ where $\mathscr{B}$ has degree $(j-k) l-$ 
$(r-t-1) \varepsilon$. Clearly, if $t \geq 2$, then $g_{m}^{\mu}$ must be composite. However, if $t=1$, then $\mu=r-2$, and $g_{m}^{\mu}$ could conceivably be simple.

Suppose that $t=1$ and that $g_{m}^{r-2}$ is simple. By 2.3.1 we may apply 1.1.4 to $g_{1}$ and $g_{2}$. As a consequence, one finds that there exist two distinct $g_{n-m}^{1}$ 's which impose two conditions on $g_{1}$, which is impossible. Therefore, we conclude that $g_{m}^{\mu}$ is always composite, possibly with base points. Therefore $X_{p}$ possesses a $g_{j l-k+\varepsilon+2}^{1}$ imposing two conditions on each of the $g_{i}$ 's. We write

$$
g_{m}^{r-t-1}=(r-t-1) g_{j l-k+\varepsilon+2}^{1}+\mathscr{B},
$$

where $\mathscr{B}$ has degree $(j-k) l-(r-t-1) \varepsilon$. We have the following

Lemma 2.3.5. If $g_{k+1}=g_{1}+g_{j l-k+\varepsilon+2}^{1}-\mathscr{D}-\mathscr{D}_{k}$, then $\mathscr{B}$ contains either $\mathscr{D}$ or $\mathscr{D}_{k}$.

Proof. The proof is exactly the same as in 2.2.1.

Corollary 2.3.6. If we are not dealing with the exceptional case for $j=4$, then $g_{i}=g_{1}+g_{T}^{1}-\mathscr{D}-\mathscr{D}_{i}$ for some divisor $\mathscr{D}$. We may combine 2.3.5, 1.2.6, and the fact that $g_{i}+g_{m}^{r-t-1}$ has dimension $3 r-2 t-2$ for $1 \leq i \leq k$, to find that $\sum_{i=k+1}^{j} \mathscr{D}_{i} \subseteq \mathscr{B}$.

Proof. Suppose that $\mathscr{B}=\mathscr{D}+\mathscr{F}$. Then

$$
g_{2}+(r-t-1) g_{T}^{1}+\mathscr{B}=\left[g_{2}+\mathscr{D}\right]+(r-t-1) g_{T}^{1}+\mathscr{F} .
$$

However, since $g_{2}+\mathscr{D}$ has dimension ${ }^{4} r+1$, the linear series $\left[g_{2}+\mathscr{D}\right]+$ $(r-t-1) g_{T}^{1}+\mathscr{F}$ has dimension at least $3 r-2 t-1$, which is a contradiction. Note that this argument is valid regardless of whether the relationships between the $g_{i}$ 's are generic or nongeneric.

\section{THE MODELS}

3.0. In this section, we derive all possible models for the generalised Castelnuovo curves discussed in $\S 2$. As we noted at the beginning of $\S 1$, if $X_{p}$ admits a single $g_{n}^{r}$, with $r \geq 6$, so that $2 g_{n}^{r}$ has minimal dimension, then any of the $g_{n}^{r}$ 's determines a birational map from $X_{p}$ into a rational normal surface scroll in $\mathbf{P}^{r}$ which will be denoted $Y$. If $X_{p}$ admits $j \geq 2 g_{n}^{r}$ 's, where $r \geq 6$, $g_{1}, \ldots, g_{j}$ satisfying the hypotheses of $\S 2$ and if $g_{i}-g_{T}^{1}$ is composite $\forall i$ then, since the hypotheses of 1.1 .19 will be satisfied, we can always map $X_{p}$ into a scroll in $\mathbf{P}^{r+2}$ using $\hat{g}_{i}=g_{i}+g_{T}^{1}$, then $\hat{g}_{i}-g_{T}^{1}$ will be simple, this fact will allow us to perform our calculations as simply as possible. The referee has remarked that instead of moving to $\mathbf{P}^{r+2}$ to perform the calculations one could note that a consequence of $g_{1}-g_{T}^{1}$ being composite is that the image of $X_{p}$ lies on a singular surface scroll in $\mathbf{P}^{r}$, then one can desingularise and work with the resulting curve.

We give a brief overview of smooth rational normal surface scrolls. References for the following material will be [8, pp. 522-527] and [2, pp. 95-100, 113-123]. A rational normal surface scroll is a ruled surface of minimal degree in $\mathbf{P}^{r}$, whose general hyperplane sections are rational normal curves of degree $r-1$. The Picard group of such a surface Pic $Y=\langle H, L\rangle$, where $H$ and

\footnotetext{
${ }^{4}$ We see this because $g_{2}+\mathscr{D}=\left[g_{1}-\mathscr{D}_{2}\right]+g_{T}^{1}$, and $\mathscr{D}_{2}$ imposes only one condition on $g_{1}$.
} 
$L$ are the classes of a hyperplane and a line (of the ruling) in $Y$, respectively. Furthermore, the intersections on $Y$ are given by

$$
L \cdot L=0, \quad L \cdot H=1, \quad H \cdot H=r-1 .
$$

These relationships allow us to calculate $\mathbf{K}_{Y}$, the divisor class of the canonical sheaf on $Y$. We know that $\mathbf{K}_{Y} \equiv T H+\beta L$ for some $T$ and $\beta$. If $C$ is a smooth curve lying in $Y$, then from the adjunction formula, the genus, $p(C)$, of $C$ is given by

$$
p(C)=1+\frac{C \cdot C+C \cdot \mathbf{K}_{Y}}{2} .
$$

Since the hyperplane sections $H$, and the line $L$, are smooth rational curves on $Y$ we can use this relationship twice to detemine that $T=-2$ and $\beta=r-3$. Note that in the special case $Y=\mathbf{P}^{2}$, where $H=L$, we have the familiar relationship $\mathbf{K}_{\mathbf{P}^{2}} \equiv-3 L$.

Consider a linear series $|T H+\beta L|$, suppose that $C \in|T H+\beta L|$. If $C$ is smooth, by combining (3.1) and (3.2) we find that

$$
p(C)=\frac{[T][T-1]}{2}(r-1)+[T-1][\beta-1] .
$$

If $C$ is of degree $n$ then $C \cdot H=n$ gives $\beta=n-T(r-1)$ and $C \cdot L=T$ shows that $C$ admits a pencil of degree $T$ which imposes two conditions on hyperplane sections. Therefore, given $X_{p}$ a generalised Castelnuovo curve of degree $n$ with a $g_{T}^{1}$ we can write down the divisor class of $\varphi\left(X_{p}\right)$ in Pic $Y$ immediately it is $T H+(n-T(r-1)) L$. If $X_{p}$ is a generalised Castelnuovo curve admitting $j, j \geq 2$, linear series of the same degree and dimension we denote its pencil by $g_{T}^{1}$. The linear series $g_{1}$ provides us with a map $\varphi: X_{p} \rightarrow Y$ where $Y$ is a rational normal surface scroll of degree $r-1$ in $\mathbf{P}^{r}$. By Theorem 1.2.1 for $2 \leq i \leq j$, there exist divisors $\mathscr{D}_{i}$ and $\mathscr{Q}_{i}$, imposing one condition on $g_{1}$ so that $g_{i}=g_{1}+g_{T}^{1}-\mathscr{D}_{i}-\mathscr{Q}_{i}$, therefore the image of $X_{p}$ under $\varphi$ is not smooth. We find that

$$
p \leq \frac{T[T-1]}{2}(r-1)+[T-1][n-T(r-1)-1]-\sum_{i} \frac{m_{i}\left(m_{i}-1\right)}{2},
$$

where the sum runs over the singularities of $\varphi\left(X_{p}\right)$ and the $m_{i}$ 's are the multiplicities of the singularities. Our task will be to find the class of, and the singularities of, the image of our curve in $Y$. To do this, we need to know either the value of $T$ or the multiplicity of one of the singular points on the image curve. We can usually glean one of these pieces of information from the residual series $g_{m}^{\mu}$.Let

$$
f(n, r, T)=\frac{T[T-1]}{2}(r-1)+[T-1][n-T(r-1)-1]
$$

it can be verified that $f(n+T, r+2, T)=f(n, r, T)$ which reflects the fact that we may work on a surface scroll in $\mathbf{P}^{r+2}$ instead of in $\mathbf{P}^{r}$. We now exhibit the classes of the curves discussed in $\S 2$.

3.1. The case $k=0, l \geq 2$. Recall that $n=(j l+2) r-(l+t)$ where $0 \leq t \leq r-1$, that

$$
l\left[g_{1}+\cdots+g_{j}\right]+g_{m}^{r-t-1}=\mathbf{K},
$$


where $m=n-(j-1) l-(j l+1) t-2$, and that

$$
p=\frac{[j l+2][j l+1]}{2} r-\frac{j l[l+1]}{2}-(j l+1) t .
$$

We discuss the following three cases:

(a) $t=0$ and $g_{m}^{r-1}$ simple.

(b) $t=0$ and $g_{m}^{r-1}$ composite.

(c) $t \geq 1$ and $g_{m}^{r-t-1}$ composite.

The calculations involved in this section and the following ones are tedious but elementary, we will provide the motivation behind each one and then present the result.

(a) Suppose that $g_{m}^{r-1}$ is simple and fixed point free, by 1.1 .4

$$
g_{1}=g_{m}^{r-1}+\mathscr{D}
$$

for some effective divisor $\mathscr{D}$. Furthermore, by 2.1 .3 ,

$$
g_{i}=g_{1}+g_{T}^{1}-\mathscr{D}-\mathscr{D}_{i} .
$$

Since

$$
l\left[g_{1}+\cdots+g_{j}\right]+g_{m}^{r-1}=\mathbf{K},
$$

and $g_{T}^{1}$ imposes two conditions upon each of the $g_{i}$ and also upon $g_{m}^{r-1}$, $T \geq j l+3$, by 2.0 .6 . We shall see that $j l+3$ is the only possible value for $T$. For the time being all we know is that

$$
\varphi\left(X_{p}\right) \in|T H+[n-T(r-1)] L|
$$

for some $T . X_{p}$ admits a unique $g_{T}^{1}$ which imposes two conditions upon $g_{1}$, and therefore, upon $g_{i}$ for $2 \leq i \leq j$. From (3.4), $p \leq f(T)$, where

$$
\begin{aligned}
f(T)= & \frac{T[T-1]}{2}(r-1)+[T-1][n-T(r-1)-1] \\
& -(j-1) \frac{[T-(j-1) l-2][T-(j-1) l-3]}{2} \\
& -\frac{[(j-1) l+2][(j-1) l+1]}{2} .
\end{aligned}
$$

We note that $f(T)$ is quadratic in $T$ and that the coefficient of $T^{2}$ is $-(r+j-2) / 2$, which is negative, therefore $f(T)$ has attains its maximum value at some $\hat{T}$. We find that

$$
\hat{T}=\frac{(2 j l+5) r+\left(2 j^{2}-4 j\right) l+5 j-8}{2 r+2 j-4} \text {. }
$$

Since

$$
|j l+3-\hat{T}|<\frac{1}{2}
$$

$f(j l+3)$ is the maximum value of $f(T)$ for $T \in \mathbf{Z}$. A futher calculation shows that $f(j l+3)=p$.

Now we must investigate the possibility that $g_{m}^{r-1}$ has fixed points. We recall Accola's lower bound on the dimension of the linear series

$$
W_{0}=l_{1} g_{n_{1}}^{r_{1}}+\cdots+l_{j} g_{n_{j}}^{r_{j}}
$$


where each $g_{n_{i}}^{r_{i}}$ is simple and $r_{i} \geq r_{k}$ if $i \leq k$ then $W_{0}$ has dimension at least

$$
\Psi\left(r_{1}, \ldots, r_{j} ; l_{1}, \ldots, l_{j}\right)=\sum_{i=1}^{j} \frac{\left(l_{i}\right)\left(l_{i}+1\right)}{2} r_{i}-\frac{\left(l_{i}\right)\left(l_{i}-1\right)}{2}+\sum_{k} \sum_{i<k} l_{i} l_{k} r_{k} .
$$

Now suppose that $g_{m}^{r-1}=g_{m-\varepsilon}^{r-1}+\mathscr{F}_{\varepsilon}^{0}$ where $\varepsilon \geq 1$, and $g_{m-\varepsilon}^{r-1}$ is fixed point free then we have

$$
l\left[g_{1}+\cdots+g_{j}\right]+g_{m-\varepsilon}^{r-1}+\mathscr{F}_{\varepsilon}^{0}=\mathbf{K} .
$$

Using Accola's estimate one can show that the series $W=l\left[g_{1}+\cdots+g_{j}\right]+g_{m-\varepsilon}^{r-1}$ has dimension at least $p-1$. Since $\varepsilon \geq 1 W$ has degree strictly less than $2 p-2$ and we have a contradiction, therefore if $g_{m}^{r-1}$ is simple it can contain no fixed points.

Therefore the only possible model for $\varphi\left(X_{p}\right) \subset Y$ when $g_{m}^{r-1}$ is simple, is one which admits a $g_{j l+3}^{1}$, and which has $j-1$ singular points of multiplicity $l+1$, and one singular point of multiplicity $(j-1) l+2$. The class of such a curve in Pic $Y$ is $[j l+3] H+[(j-1) l+3-r] L$.

(b) Let us suppose that $g_{m}^{r-1}$ is composite. Since $m=(r-1)(j l+2)$, we deduce, from 1.1.5, that $T=j l+2$. Thus, the class of $\varphi\left(X_{p}\right)$ in Pic $Y$ is $[j l+2] H+[(j-1) l+2] L$. Apart from one case when $j=4$, we have seen in $\S 1$ that if $i \geq 2$ there exist divisors $\mathscr{D}$ and $\mathscr{D}_{i}$, of degree $x$ and $T-x$ respectively, each lying in some divisor of $g_{j l+2}^{1}$, so that $g_{i}=g_{1}+g_{j l+2}^{1}-$ $\mathscr{D}-\mathscr{D}_{i}$. Our aim now is to calculate all possible values of $x$. We shall consider the exceptional case for $j=4$ later. We have the inequality, $p \leq$ $[j l+2][j l+1] r / 2+f(x)$ where

$$
\begin{aligned}
f(x)= & {[j l+1][(j-1) l+1]-\frac{[j l+2][j l+1]}{2} } \\
& -(j-1) \frac{[j l+2-x][j l+1-x]}{2}-\frac{x[x-1]}{2} .
\end{aligned}
$$

The coefficient of $x^{2}$ in $f(x)$ is $-j / 2$ so $f(x)$ has a maximum at some point $\hat{x}$. We find that

$$
\hat{x}=\frac{\left(2 j^{2}-2 j\right) l+3 j-2}{2 j}
$$

Since

$$
|(j-1) l+1-\hat{x}|<1 / 2
$$

$f(x)$, for $x \in \mathbf{Z}$, attains its maximum value at $x=(j-1) l+1$. To finish off one shows that

$$
f(j l-l+1)=-[j l][l+1] / 2 .
$$

Thus,

$$
p \leq f(j l-l+1)+[j l+2][j l+1] r / 2=p,
$$

and we have exhibited a model for $X_{p}$ in $\mathbf{P}^{r}$ whose class in Pic $Y$ is $[j l+2] H+[(j-1)+2] L$. The model possesses $j$ singular points, $j-1$ of multiplicity $l+1$, and one of multiplicity $j l-l+1$.

The exceptional case $j=4$. In this case there exist divisors $\mathscr{D}, \mathscr{E}$, and $F$, all of degree $2 l+1$, and all lying in different divisors of $g_{4 l+2}^{1}$, so that $g_{2}=g_{1}+g_{j l+2}^{1}-\mathscr{D}-\mathscr{E}, g_{3}=g_{1}+g_{j l+2}^{1}-\mathscr{D}-\mathscr{F}$, and $g_{4}=g_{1}+g_{j l+2}^{1}-\mathscr{E}-\mathscr{F}$. 
The existence of such a model for $X_{p}$ is easily verifiable directly from (3.4). The class of $\varphi\left(X_{p}\right)$ in Pic $Y$ is $(4 l+2) H+(3 l+2) L$, and the curve has three singularities of multiplicity $2 l+1$, no two of which are contained in the same line of the ruling on $Y$.

(c) Now we must consider possible models for the case $n=(j l+2) r-$ $(l+t)$ where $1 \leq t \leq r-1$. By 1.1 .5 , since $g_{m}^{r-t-1}$ is composite and $m=$ $(r-t-1)(j l+2)$, there exists a $g_{j l+2}^{1}$ which imposes two conditions on each $g_{i}$. All we have to do is to produce the correct singularities and we are done. The calculations go ahead as before. The only model is one whose image in $\mathbf{P}^{r}$ has class $(j l+2) H+(j l-l-t+2) L$ in Pic $Y$, and which possesses $j$ singular points, $j-1$ of which have multiplicity $l+1$, and one of which has multiplicity $(j-1) l+1$. If $t=r-1$ then we do not get any information about the $g_{T}^{1}$ from $g_{m}^{\mu}$, however from 2.0 .6 we see that $T \geq j l+2$ and $T>j l+2$ will be excluded by arguing as in 3.2 .1 below.

Similarly, in the exceptional case when $j=4$, we obtain a curve whose class in Pic $Y$ is $(4 l+2) H+(3 l+2-t) L$ with three singularities of multiplicity $2 l+1$.

3.2. The case $k=1$. In this case we have $n=(j l+1) r-(l+t)$, where $0 \leq t \leq r-2$. We write $\mathbf{K}$, the canonical series as

$$
[l-1] g_{1}+l\left[g_{2}+\cdots+g_{j}\right]+g_{m}^{r-t-2}=\mathbf{K},
$$

where $m=n-(j l+2)-j l t$, and

$$
p=\frac{[j l][j l+1]}{2} r-\frac{[j l][l+1]}{2}-j l t .
$$

We consider the following three cases:

(a) $t=0, g_{m}^{r-2}$ is simple.

(b) $t=0, g_{m}^{r-2}$ is composite, and $g_{m}^{r-2}=(r-2) g_{j l+\varepsilon+1}^{1}+\mathscr{B}$, where $\mathscr{B}$ is the fixed point set of $g_{m}^{r-2}$. $\mathscr{B}$ has degree $(j-1) l-\varepsilon(r-2)$. We shall show that $\varepsilon=0$ is the only possibility.

(c) $t \geq 1$, and $g_{m}^{r-2-t}=(r-2-t) g_{j l+\varepsilon+1}^{1}+\mathscr{B}$, where $\mathscr{B}$ is the fixed point set of $g_{m}^{r-2-t}$. $\mathscr{B}$ has degree $(j-1) l-(r-t-2) \varepsilon$. We shall show that $\varepsilon=0$ is the only possibility.

(a) If $g_{m}^{r-2}$ is fixed point free then there exists a $g_{j l+2}^{1}$ so that $g_{1}=g_{m}^{r-2}+$ $g_{j l+2}^{1}$. Suppose that we are not in the exceptional case. There exists a divisor $\mathscr{D}$, so that for $2 \leq i \leq j, g_{i}=g_{1}+g_{j l+2}^{1}-\mathscr{D}-\mathscr{D}_{i}$. Let $\mathscr{D}$ have degree $x$. From (3.4), $p \leq(j l+1)(j l) r / 2+f(x)$, where

$$
\begin{aligned}
f(x)= & {[j l+1][j l-l+1]-\frac{[j l+2][j l+1]}{2}-\frac{[x][x-1]}{2} } \\
& -(j-1) \frac{[j l+2-x][j l+1-x]}{2} .
\end{aligned}
$$

We note, as before, that $f(x)$ is quadratic in $x$ with negative $x^{2}$ coefficient, its maximum is attained at

$$
\hat{x}=\left(\left(2 j^{2}-2 j\right) l+3 j-2\right) / 2 j .
$$

Since

$$
|(j-1) l+1-\hat{x}|=|(2-j) / 2 j|<1 / 2,
$$


$f(x)$ attains its maximum value over the integers at $(j-1) l+1$. A calculation shows that

$$
f(j l-l+1)=-[j l][l+1] / 2 .
$$

Therefore,

$$
p \leq \frac{[j l+1][j l]}{2} r-\frac{[j l][l+1]}{2}=p
$$

and so we have exhibited a model for $X_{p}$, whose image in $\mathbf{P}^{r}$ has class $(j l+2) H+(j l+2-l-r) L$ in Pic $Y$. Our model has $j-1$ singularities of multiplicity $l+1$, and one singular point of multiplicity $j l-l+1$.

If $g_{m}^{r-2}$ has base points then $X_{p}$ admits a $g_{j l+2+\varepsilon}^{1}$ where $\varepsilon \geq 1$. One can show using the same argument as the one given above that no such model exists.

The exceptional case $j=4$. If $j=4$ then it is possible that there are divisors $\mathscr{D}, \mathscr{E}$, and $\mathscr{F}$, each of degree $2 l+1$ so that $g_{2}=g_{1}+g_{4 l+2}^{1}-\mathscr{D}-\mathscr{E}, g_{3}=$ $g_{1}+g_{4 l+2}^{1}-\mathscr{D}-\mathscr{F}$, and $g_{4}=g_{1}+g_{4 l+2}^{1}-\mathscr{E}-\mathscr{F}$. Once again, it can be verified directly from (3.4) that there is such a model. Therefore, if $j=4$ and $g_{n-j l-2}^{r-2}$ is simple, we can exhibit a model for $X_{p}$ which has class $(4 l+2) H+(3 l+2-r) L$ in Pic $Y$, and three singularities of multiplicity $2 l+1$.

(b) $g_{m}^{r-2}=(r-2) g_{j l+\varepsilon+1}^{1}+\mathscr{B}$, where $\mathscr{B}$ is the fixed point set of $g_{m}^{r-2} . \mathscr{B}$ has degree $(j-1) l-\varepsilon(r-2)$. First, let us suppose that $\varepsilon=0$.

If $j \neq 4$, there exists a divisor $\mathscr{D}$ of degree $x$, which lies in some divisor of $g_{j l+1}^{1}$ so that, for $2 \leq i \leq j, g_{i}=g_{1}+g_{j l+1}^{1}-\mathscr{D}-\mathscr{D}_{i}$. From (3.4)

$$
p \leq(j l)(j l+1) r / 2+f(x) \text {, }
$$

where

$$
f(x)=[j l][j l-l]-\frac{[j l+1][j l]}{2}-(j-1) \frac{[j l+1-x][j l-x]}{2}-\frac{[x][x-1]}{2} .
$$

$f(x)$ is quadratic in $x$ with negative leading coefficient. It assumes its maximum value at

$$
\hat{x}=(j-1) l+\frac{1}{2}
$$

which implies that $f(j l-l)$ and $f(j l-l+1)$ are the maximum values of $f(x)$ when $x$ is restricted to the integers. One shows that

$$
f(j l-l)=f(j l-l+1)=-[j l][l+1] / 2 .
$$

Thus,

$$
p \leq \frac{[j l+1][j l]}{2} r-\frac{[j l][l+1]}{2}=p .
$$

and so we have two distinct models for $X_{p}$. Both have class $(j l+1) H+$ $(j l-l+1) L$ in Pic $Y$, however, one has $j-1$ singular points of multiplicity $l$ and one singular point of multiplicity $(j-1) l+1$, while the other has $j-1$ singular points of multiplicity $l+1$, and one singular point of multiplicity $(j-1) l$.

(c) If $t \geq 1$, then $g_{m}^{r-t-2}$ is composite and is compounded of a $g_{j l+\varepsilon+1}^{1}$ (from 2.0.6 $T \geq j l+1$ ), and

$$
p=\frac{[j l+1][j l]}{2} r-\frac{[j l][l+1]}{2}-j l t .
$$

If we assume that $\varepsilon=0$, then the calculation in (b) above shows that there exist two models for $X_{p}$, both models for $X_{p}$ have class $(j l+1) H+(j l-l+1-t) L$ 
in Pic $Y$, one has $j-1$ singularities of multiplicity $l$ and one singular point of multiplicity $(j-1) l+1$, the other has $j-1$ singular points of multiplicity $l+1$ and one singular point of multiplicity $(j-1) l$.

Lemma 3.2.1. The models derived in (b) and (c) are the only possible ones for $X_{p}$, i.e., if $\mathbf{K}-\left[(l-1) g_{1}+l\left(g_{2}+\cdots+g_{j}\right)\right]$ is composite, then there exist no models for $X_{p}$ admitting a $g_{j l+\varepsilon+1}^{1}$ which imposes two conditions on each $g_{i}$ with $\varepsilon \geq 1$.

Proof.

Part 1. Suppose that $n=(j l+1) r-l$, that $\varepsilon \geq 1$, and that we are not in the exceptional case for $j=4$. Then for some divisor $\mathscr{D}$, of degree $x$, contained in a divisor of $g_{j l+\varepsilon+1}^{1}$ we have $g_{i}=g_{1}+g_{j l+\varepsilon+1}^{1}-\mathscr{D}-\mathscr{D}_{i}$, and the inequality $p \leq f_{1}(x, \varepsilon)$, where

$$
\begin{aligned}
f_{1}(x, \varepsilon)= & \frac{[j l+\varepsilon+1][j l+\varepsilon]}{2}(r-1)+[j l+\varepsilon][j l-l-\varepsilon(r-1)] \\
& -(j-1) \frac{[j l+\varepsilon+1-x][j l+\varepsilon-x]}{2}-\frac{[x][x-1]}{2} .
\end{aligned}
$$

$f_{1}(x, \varepsilon)$ attains its maximum value, as a function of $x$, at

$$
\hat{x}=\frac{(j-1)(2 j l+2 \varepsilon+1)+1}{2 j}=(j-1) l+\varepsilon+\frac{1}{2}-\frac{\varepsilon}{j} .
$$

From (2.2.2), either $\mathscr{D} \subset \mathscr{B}$ or $\sum_{i=2}^{j} \mathscr{D}_{i} \subset \mathscr{B}$. Since $\mathscr{B}$ has degree $(j-1) l-(r-2) \varepsilon$, and the degree of $\mathscr{D}_{i}$ is $j l+\varepsilon+1-x$, we have

$$
x \leq x_{0}=(j-1) l-(r-2) \varepsilon \text { if } \mathscr{D} \subset \mathscr{B},
$$

or

$$
x \geq x_{1}=(j-1) l+\varepsilon+1 \quad \text { if } \sum_{i=2}^{j} \mathscr{D}_{i} \subset \mathscr{B} .
$$

Note that these two values of $x$ are, respectively, greater than and less than $\hat{x}$. Therefore, if we can show that $f_{1}\left(x_{0}, \varepsilon\right)$ and $f_{1}\left(x_{1}, \varepsilon\right)$ are both less than $p$ when $\varepsilon \geq 1$, our proof will be complete. By observing that $x_{0} \leq j l-l$, and that $j l-l+\varepsilon \leq x_{1}$ we can solve the problem by showing that $f_{1}(j l-l, \varepsilon)$ and $f_{1}(j l-l+\varepsilon+1, \varepsilon)$ are both less than $p$.

Omitting the details, one finds that

$$
f_{1}(j l-l, \varepsilon)=p-\frac{\varepsilon(\varepsilon-1)}{2} r-\frac{(j-2) \varepsilon^{2}+j \varepsilon}{2}
$$

and

$$
f_{1}(j l-l+\varepsilon+1, \varepsilon)=p-\frac{\varepsilon(\varepsilon-1)}{2} r-\varepsilon .
$$

Clearly if $\varepsilon \geq 1$ both quantities are smaller than $p$.

Part 2. Finally, we must consider the case $n=(j l+1) r-(l+t)$, where $1 \leq t \leq r-2$. Recall that we have

$$
(l-1) g_{1}+l\left[g_{2}+\cdots+g_{j}\right]+(r-t-2) g_{j l+\varepsilon+1}^{1}+\mathscr{B}=\mathbf{K},
$$

where $\mathscr{B}$ has degree $(j-1) l-(r-2-t) \varepsilon$. We shall show that there is no model for $X_{p}$ if $\varepsilon \geq 1$. Unless we are in the exceptional case for $j=4$, there 
exists a divisor $\mathscr{D}$ of degree $x$, lying in some divisor of $g_{j l+\varepsilon+1}^{1}$, so that for $2 \leq i \leq j$

$$
g_{i}=g_{1}+g_{j l+\varepsilon+1}^{1}-\mathscr{D}-\mathscr{D}_{i} .
$$

As above, $p \leq f_{2}(x, \varepsilon)$, where

$$
\begin{aligned}
f_{2}(x, \varepsilon)= & \frac{[j l+\varepsilon+1][j l+\varepsilon]}{2}(r-1)+[j l+\varepsilon][(j-1) l-\varepsilon(r-1)-t] \\
& -\frac{[x][x-1]}{2}-(j-1) \frac{[j l+\varepsilon+1-x][j l+\varepsilon-x]}{2} \\
= & f_{1}(x, \varepsilon)-(j l+\varepsilon) t .
\end{aligned}
$$

As a function of $x$ the left-hand side attains its maximum value at

$$
\hat{x}=(j-1) l+\varepsilon+1 / 2-\varepsilon / j .
$$

However, since either $\mathscr{D} \subset \mathscr{B}$ or $\sum \mathscr{D}_{i} \subset \mathscr{B}$, by 2.1.4, either

$$
x \leq x_{0}=(j-1) l-(r-t-2) \varepsilon
$$

or

$$
(j-1)(j l+\varepsilon+1-x) \leq(j-1) l-(r-t-2) \varepsilon .
$$

The second inequality implies that

$$
x \geq x_{1}=(j-1) l+\varepsilon+1+\delta
$$

where $\delta \geq 0$. Once again, since

$$
x_{0} \leq(j-1) l \leq \hat{x} \leq(j-1) l+1+\varepsilon \leq x_{1}
$$

it is enough to show that $f_{2}(j l-l, \varepsilon)<p$ and $f_{2}(j l-l+1+\varepsilon, \varepsilon)<p$. We know from our earlier calculations with $f_{1}(x, \varepsilon)$ that both of these inequalities hold.

The exceptional case $j=4 . X_{p}$ admits a $g_{4 l+\varepsilon+1}^{1}$ imposing two conditions on each $g_{i}$ and $\mathscr{B}$ has degree $3 l-(r-t-2) \varepsilon$. Suppose that there exist divisors $\mathscr{D}, \mathscr{E}$, and $\mathscr{F}$, all of the same degree $d \geq 2 l$, so that $g_{2}=g_{1}+g_{4 l+\varepsilon+1}^{1}-$ $\mathscr{D}-\mathscr{E}, g_{3}=g_{1}+g_{4 l+\varepsilon+1}^{1}-\mathscr{D}-\mathscr{F}$, and $g_{4}=g_{1}+g_{4 l+\varepsilon+1}^{1}-\mathscr{E}-\mathscr{F}$. Since $g_{i}+g_{m}^{r-t-2}$ has dimension $3 r-2 t-3$, for $2 \leq i \leq 4$, we see by Lemma 2.2 .1 that two of $\{\mathscr{D}, \mathscr{E}, \mathscr{F}\}$ lie in $\mathscr{B}$. However, by counting degrees we find that this is impossible, and we conclude that there is no model for $X_{p}$.

3.3. The case $k \geq 2$. We have $j$ linear series of dimension $r$ and degree $n$. We have seen before that $n=(j l-k+2) r-(l+t-1)$, where $k \in \mathbf{Z}$, $2 \leq k \leq j-1$, and, $0 \leq t \leq r-1$. Then, we can write

$$
(l-1)\left[g_{1}+\cdots+g_{k}\right]+l\left[g_{k+1}+\cdots+g_{j}\right]+g_{m}^{r-t-1}=\mathbf{K},
$$

where $m=n-(k-1)(l-1)-(j l-k+1) t-2$ and

$$
p=\frac{[j l-k+2][j l-k+1]}{2} r-\frac{[j l][l-1]}{2}-(j l-k+1) t .
$$

Aside from the exceptional case, which we shall discuss on an ad hoc basis, the following are the possibilities:

(a) $t=0$ and $g_{m}^{r-1}$ is simple, with $X_{p}$ admitting a $g_{T}^{1}$ which imposes two conditions on each $g_{i}$ and $g_{m}^{r-1}$. 
(b) $t=0$ and $g_{m}^{r-1}$ is composite and equal to $(r-1) g_{j l-k+2+\varepsilon}^{1}+\mathscr{B}$, where $\mathscr{B}$ has dimension zero and degree $(j-k) l-(r-1) \varepsilon$. We shall see in this section that $\varepsilon=0$ is the only permissible value for $\varepsilon$. Note that $g_{j l-k+\varepsilon+2}^{1}$ imposes two conditions on each $g_{i}$.

(c) $t \geq 1$ and $g_{m}^{r-t-1}=(r-t-1) g_{j l-k+2+\varepsilon}^{1}+\mathscr{B}$, where $\mathscr{B}$ has degree $(j-k) l-(r-t-1) \varepsilon$. We shall see, as in (b), that $\varepsilon=0$ is the only case which can occur.

(a) First suppose that $g_{m}^{r-1}$ is simple and fixed point free. Recall, from 2.3.4, if $j \geq 5$ and $k \geq 3$, then $g_{m}^{r-1}$ cannot be simple. Suppose that $j \geq 3$, $k=2$ and $X_{p}$ admits a $g_{T}^{1}$. Then there exists a divisor $\mathscr{D}_{2}$, of degree $l+1$, so that $g_{1}=g_{m}^{r-1}+\mathscr{D}_{2}$ and $g_{2}=g_{1}+g_{T}^{1}-\mathscr{D}-\mathscr{D}_{2}$. From 2.3.4 we see that for $3 \leq i \leq j$

$$
g_{i}=g_{1}+g_{T}^{1}-\mathscr{D}-\mathscr{D}_{i} .
$$

From (3.4) $p \leq f(T)$, where

$$
\begin{aligned}
f(T)= & \frac{[T][T-1]}{2}(r-1)+[T-1][n-T(r-1)-1] \\
& -(j-1) \frac{[l+1][l]}{2}-\frac{[T-l-1][T-l-2]}{2} .
\end{aligned}
$$

$f(T)$ assumes its maximum value at

$$
\hat{T}=(2 j l+1) r+2 / 2 r .
$$

We note that

$$
|j l+1-\hat{T}|=\left|\frac{1}{2}-\frac{1}{r}\right|<\frac{1}{2}
$$

and conclude that $f(T)$ attains its maximum over the integers at $j l+1$. A computation verifies that $f(j l+1)=p$ and we have a model for $X_{p}$ in $\mathbf{P}^{r}$, whose class in Pic $Y$ is $(j l+1) H+(j l-l-r+2) L$. Our model has $j-1$ singular points of multiplicity $l+1$, and one singular point of multiplicity $j l-l$.

The exceptional case $j=4$. Suppose that there are three divisors, $\mathscr{D}, \mathscr{E}$, and $\mathscr{F}$, all of the same degree, each lying in a different divisor of $g_{T}^{1}$, so that $g_{2}=g_{1}+g_{T}^{1}-\mathscr{D}-\mathscr{E}, g_{3}=g_{1}+g_{T}^{1}-\mathscr{D}-\mathscr{F}$, and $g_{4}=g_{1}+g_{T}^{1}-\mathscr{E}-\mathscr{F}$. If $k=2$, then as we saw above, one of the divisors $\mathscr{D}$ or $\mathscr{E}$, has degree $l+1$. But then, $T=2 l+2<4 l+1$, so this case cannot arise.

If $k=3$, then $\mathscr{D}$ has degree $2 l$. Then, $T=4 l$ and our usual calculation exhibits a model for $X_{p}$ whose image in $\mathbf{P}^{r}$ has class $4 l H+(3 l-r+1) L$ in Pic $Y$ which possesses three singular points of multiplicity $2 l$.

Now we briefly mention the case where the residual series, although simple, possesses fixed points. Suppose that

$$
(l-1)\left[g_{1}+\cdots+g_{k}\right]+l\left[g_{k+1}+\cdots+g_{j}\right]+g_{m+\varepsilon}^{r-1}=\mathbf{K},
$$

where $g_{m+\varepsilon}^{r-1}=g_{m}^{r-1}+\mathscr{F}_{\varepsilon}^{0}$ where $\varepsilon \geq 1$ and $g_{m}^{r-1}$ is simple and fixed point free. One can show using Accola's arguments that, for $k+1 \leq i \leq j$, the series $g_{i}+g_{m}^{r-1}$ has dimension $3 r-1$, therefore 2.3.3 and 2.2.4 apply and a repitition of the calculations given above show that no such model can exist if $\varepsilon \geq 1$.

(b) Let us suppose that $\varepsilon=0$. Leaving aside for one moment the complications involved if $j=4$, we know of the existence of a divisor $\mathscr{D}$ of 
degree $x$ which imposes one condition on $g_{1}$ so that, for $2 \leq i \leq j, g_{i}=$ $g_{1}+g_{j l-k+2}^{1}-\mathscr{D}-\mathscr{D}_{i}$. From $(3.4), p \leq f(x, \varepsilon)$ where

$$
\begin{aligned}
f(x, \varepsilon)= & \frac{[j l-k+2][j l-k+1]}{2}(r-1)+[j l-k+1][j l-l-k+2] \\
& -\frac{(j-1)[j l-k+2-x][j l-k+1-x]}{2}-\frac{[x][x-1]}{2} .
\end{aligned}
$$

$f(x, \varepsilon)$ attains its maximum value at

$$
\hat{x}=\frac{(j-1)(2 j l-2 k+3)+1}{2 j}=(j-1) l-k+2-\frac{j-2 k+2}{2 j} .
$$

Since $2 \leq k \leq j-1$,

$$
|j l-k-l+2-\hat{x}|=|(j-2 k+2) / 2 j|<1 / 2 .
$$

Therefore, $f(x, \varepsilon)$ restricted to the integers attains its maximum value at $(j-1) l-k+2$, one can verify that $f(j l-l-k+2)=p$. Thus, we can exhibit a model for $X_{p}$ whose image in $\mathbf{P}^{r}$ has class $[j l-k+2] H+[j l-l-k+3] L$ in Pic $Y$. The image of the curve in $\mathbf{P}^{r}$ has $j$ singular points, $j-1$ of which have multiplicity $l$, and one of which has multiplicity $(j-1) l-k+2$.

The exceptional case $j=4$. If $k=3$, and $\varepsilon=0$, then $j l-k+2$ is not divisible by 2 , and so the situation in (b) is the only possible one. However, if $k=2$ then we can exhibit a model for $X_{p}$ whose image in $\mathbf{P}^{r}$ has class $4 l \mathrm{H}+$ $(3 l+1) L$ in Pic $Y$ and which possesses three singular points of multiplicity $2 l$.

(c) Finally, we consider the case $n=(j l-k+2) r-(l+t-1)$ where $1 \leq t \leq r-1$. In this case

$$
(l-1)\left[g_{1}+\cdots+g_{k}\right]+l\left[g_{k+1}+\cdots+g_{j}\right]+(r-t-1) g_{j l-k+2+\varepsilon}^{1}+\mathscr{B}=\mathbf{K} .
$$

If we assume that $\varepsilon=0$, the calculations performed in (b) show that there is a model for $X_{p}$ and we do not repeat them here. The class of the image of $X_{p}$ in Pic $Y$ is $(j l-k+2) H+(j l-k-l+3-t) L$. The singularities of the image of $X_{p}$ in $\mathbf{P}^{r}$ are the same as those exhibited in (b), namely $j-1$ of multiplicity $l$, and one of multiplicity $(j-1) l-k+2$.

Lemma 3.3.1. If $\mathbf{K}-\left[(l-1)\left(g_{1}+\cdots+g_{k}\right)+l\left(g_{k+1}+\cdots+g_{j}\right)\right]$ is composite, then the models derived above are the only possible ones for $X_{p}$, i.e., there exist no models for $X_{p}$ admitting a $g_{j l-k+2+\varepsilon}^{1}$ which imposes two conditions on each $g_{i}$ with $\varepsilon \geq 1$.

Proof. We shall argue in exactly the same fashion as before. Let us suppose that we are in the nonexceptional case, then there exists a divisor $\mathscr{D}$, of degree $x$, so that $g_{i}=g_{1}+g_{j l-k+\varepsilon+2}^{1}-\mathscr{D}-\mathscr{D}_{i}, 2 \leq i \leq j$. We have the inequality $p \leq f(x, \varepsilon)$, where

$$
\begin{aligned}
f(x, \varepsilon)= & \frac{[j l-k+\varepsilon+2][j l-k+\varepsilon+1]}{2}(r-1)+[j l-k+\varepsilon+1] \\
& \cdot[(j l-k+2) r-(l+t-1)-(j l-k+\varepsilon+2)(r-1)-1] \\
& -(j-1) \frac{[j l-k+\varepsilon+2-x][j l-k+\varepsilon+1-x]}{2}-\frac{[x][x-1]}{2} .
\end{aligned}
$$


We find that $f(x, \varepsilon)$ achieves its maximum value, as a function of $x$, at

$$
\begin{aligned}
\hat{x} & =\frac{(j-1)(2 j l-2 k+2 \varepsilon+3)+1}{2 j} \\
& =(j-1) l-k+\varepsilon+2+\frac{k}{j}-\frac{1}{2}-\frac{\varepsilon+1}{j} .
\end{aligned}
$$

Let $x_{0}=(j-1) l-k+\varepsilon+2$. Since $\varepsilon \geq 1$ and $2 \leq k \leq j$, which implies that $|k / j-1 / 2| \leq 1 / 2$, we may conclude that $\hat{x}<x_{0}+1 / 2$.

Because $k \geq 2$, we know from 2.3.6 that $\sum_{i=k+1}^{j} D_{i} \subset \mathscr{B}$, where $\mathscr{B}$ has degree $(j-k) l-(r-t-1) \varepsilon$, therefore

$$
(j-k)(j l-k+\varepsilon+2-x) \leq(j-k) l-(r-t-1) \varepsilon .
$$

The last inequality implies that $x \geq(j-1) l-k+\varepsilon+2+\delta=x_{0}+\delta \geq[\hat{x}]$, since $\delta \geq 0$. There are two cases to consider:

(i) If $1 \leq t \leq r-1$, it will be enough to show that $f\left(x_{0}, \varepsilon\right)<p, \forall \varepsilon \geq 1$.

(ii) If $t=0$ it will be enough to show that $f\left(x_{0}+1, \varepsilon\right)<p, \forall \varepsilon \geq 1$.

One finds that

$$
\begin{aligned}
& \text { (i) } f\left(x_{0}, \varepsilon\right)=p-\frac{\varepsilon(\varepsilon-1)}{2} r-t \varepsilon \\
& \text { (ii) } f\left(x_{0}+1, \varepsilon\right)=p-(j-k)-\frac{\varepsilon(\varepsilon-1)}{2} r-\varepsilon,
\end{aligned}
$$

which completes our analysis of the nonexceptional cases.

The exceptional case $j=4$. If a model exists and $X_{p}$ possesses a $g_{4 l-k+\varepsilon+2}^{1}$, then we know immediately that $X_{p}$ must have divisors $\mathscr{D}, \mathscr{E}$, and $\mathscr{F}$ all of the same degree, and lying in different divisors of $g_{4 l-k+\varepsilon+2}^{1}$, so that $g_{2}=g_{1}+$ $g_{4 l-k+\varepsilon+2}^{1}-\mathscr{D}-\mathscr{E}, g_{3}=g_{1}+g_{4 l-k+\varepsilon+2}^{1}-\mathscr{D}-\mathscr{F}$, and $g_{4}=g_{1}+g_{4 l-k+\varepsilon+2}^{1}-\mathscr{E}-\mathscr{F}$. If $k=2$, then $\varepsilon=2 \rho$ and $\mathscr{F} \subseteq \mathscr{B}$ (Since $g_{2}+g_{m}^{\mu}$ has dimension $3 r-2 t-2$, neither $\mathscr{D}$ nor $\mathscr{E}$ can be contained in $\mathscr{B})$ where $\mathscr{B}$ is the fixed divisor of the residual series. However, $\mathscr{B}$ has degree $2 l-(r-t-1) \varepsilon$, while $\mathscr{D}, \mathscr{E}$, and $\mathscr{F}$ all have degree $2 l+\rho$, which is a contradiction. If $k=3$, then one of $\mathscr{E}, \mathscr{F}$ is contained in $\mathscr{B}$, which has degree less than $l$. Counting degrees leads to a contradiction.

\section{THE PLANE MODELS}

We choose (generic) points $\left\{x_{1}, \ldots, x_{r-2}\right\}$ on $X_{p}$, so that no two points lie in the same divisor of $g_{T}^{1}$. Then consider the series

$$
g_{n-r+2}^{2}=g_{1}-\left[x_{1}+\cdots+x_{r-2}\right] \text {. }
$$

Since $g_{1}$ is complete, we observe that $g_{n-r+2}^{2}$ is also complete and setting $d=n-r+2, g_{d}^{2}$ provides us with a plane model, $\mathscr{C}_{d} \subset \mathbf{P}^{2}$ for $X_{p}$ via the map $\pi: X_{p} \mapsto \mathscr{C}_{d}$ where $\pi$ is the birational map composed of a series of projections through the points $x_{1}, \ldots, x_{r-2}$. We note that $\mathscr{C}_{d}$ has at least $r-2$ singularities of multiplicity $T-1$, since each time we project through $x_{i}$ the $T-1$ other points in the divisor of $g_{T}^{1}$ are mapped to one point in $\mathbf{P}^{2}$. Since $g_{T}^{1}$ imposes two conditions on $g_{1}$, it imposes two conditions on $g_{d}^{2}$, i.e., 


$$
g_{d}^{2}=g_{T}^{1}+g_{d-T}^{0},
$$

which indicates the existence of a singular point of multiplicity $d-T$ on $\mathscr{C}_{d}$. All of the models possessing a $g_{T}^{1}$ in $\mathbf{P}^{r}$ which we exhibited in $\S 3$ are transformed into plane models of degree $d$. These plane models possess, in addition to the singular points exhibited in $\S 3, r-2$ singular points of multiplicity $T-1$ and one singular point of multiplicity $d-T$. Let $R_{1}, \ldots, R_{r-2}$ be the $r-2$ singular points on $C_{d}$ of multiplicity $T-1$, let $Q$ be the singular point of multiplicity $d-T$ on $C_{d}$, let $D=\pi(\mathscr{D})$ and $D_{i}=\pi\left(\mathscr{D}_{i}\right)$ where $\mathscr{D}$ and $\mathscr{D}_{i}$ are as in $\S 3$.

In general then, the plane model for a generalized Castelnuovo curve has degree $d=n-r-2$ and $j+r-2$ singular points ${ }^{5}$. We note that we can eliminate $r-2$, (if $r$ is even) or, $r-3$ (if $r$ is odd), of the singular points by performing a succession of quadratic transformations centered at $Q$ and two of the $R_{i}$ 's.

These quadratic transformations will only eliminate the singularities at the $R_{i}$ 's if they are in general position. If this is the case, and we assume that $r$ is even, and that $j=2$, then we arrive at the same model by subtracting off $(r-2) / 2$ multiples of $g_{T}^{1}$ from $g_{n}^{r}$. Then, we are left with a $g_{d}^{2}$, which has three singularities, $Q$ which is of multiplicity $d-T, P$ which is of multiplicity $s$ and $D_{1}$ which is of multiplicity $T-s$. Such a model may be constructed by taking a homogeneous polynomial $F(X, Y, Z)$ of degree $d$ in $\mathbf{P}^{2}$ and requiring the requisite singular points to be at the points $[1,0,0],[0,1,0]$, and $[0,0,1]$.

If the $R_{i}$ are in general position, and if we assume that $r$ is odd and that $j=2$, then we arrive at a model by subtracting off $(r-3) / 2$ multiples of $g_{T}^{1}$ from $g_{n}^{r}$ to obtain $g_{d^{\prime}}^{3}$. If we subtract off one more point, say $x$, then we have a $g_{d}^{2}$ which has four singular points, $Q$ which has multiplicity $d-T, P$ which has multiplicity $s, D_{1}$ which has multiplicity $T-s$ and $R$ which has multiplicity $T-1$. If we take a quadratic transformation centered at $Q, P$ and $R$ then, since $Q$ has degree $d-T$ and $R$ has degree $T-1$, the singularity formerly at $P$ will vanish. Now we can construct the curve in $\mathbf{P}^{2}$ as before.

For $j \geq 3$ linear series the existence of plane models for generalised Castelnuovo curves is not quite so clear, since, in general it will involve constructing a plane model of degree $d$ with $j$ singular points. We shall not discuss this problem any further except to note that there are (unique) models for all of the Riemann surfaces discussed in $\S 5$.

4.1. A summary of the models. Tables 4.1.1-4.1.4 summarise the classification of models for generalised Castelnuovo curves performed in $\S 3$. Our notation is as follows: $\mu$ is the dimension of the residual linear series, $T$ is the degree of the $g_{T}^{1}$, and $S$ is the multiplicity of the largest singular point on the image curve in projective space. There are $j-1$ other singular points of multiplicity $T-S$ (apart from the exceptional case $j=4$ when there are three singular points of multiplicity $T / 2$ ).

\footnotetext{
${ }^{5}$ Except when $j=4$ and there are $r-2+3$ singular points.
} 
TABLE 4.1.1

\begin{tabular}{|c|c|c|}
\hline \multicolumn{2}{|c|}{ Case $k=0: n=(j l+2) r-(l+t), l \geq 2$} \\
\hline$\mu$ & $T$ & $S$ \\
\hline$r-t-1$ & $j l+2$ & $(j-1) l+1$ \\
\hline$r-1^{*}$ & $j l+3$ & $(j-1) l+2$ \\
\hline
\end{tabular}

*(Residual simple.)

TABLE 4.1.2

\begin{tabular}{|c|c|c|}
\hline \multicolumn{3}{|c|}{ Case $k=1: n=(j l+1) r-(l+t)$} \\
\hline$\mu$ & $T$ & $S$ \\
\hline$r-t-2$ & $j l+1$ & $(j-1) l$ \\
\hline$r-t-2$ & $j l+1$ & $(j-1) l+1$ \\
\hline$r-2^{*}$ & $j l+2$ & $(j-1) l+1$ \\
\hline
\end{tabular}

*(Residual simple.)

TABLE 4.1.3

\begin{tabular}{|c|c|c|c|}
\hline \multicolumn{4}{|c|}{ The case $k \geq 2 \& \quad j \geq 5: n=(j l-k+2) r-(l+t-1)$} \\
\hline Value of $k$ & $\mu$ & $T$ & $S$ \\
\hline 2 & $r-1^{*}$ & $j l+1$ & $(j-1) l$ \\
\hline$\geq 2$ & $r-t-1$ & $j l-k+2$ & $(j-1) l-k+2$ \\
\hline
\end{tabular}

*(Residual simple.)

TABLE 4.1.4

\begin{tabular}{|c|c|c|c|}
\hline \multicolumn{4}{|c|}{ The exceptional case $j=4$} \\
\hline Value of $k$ & $\mu$ & $T$ & $S$ \\
\hline 0 & $r-t-1$ & $4 l+2$ & $2 l+1$ \\
\hline 1 & $r-t-1$ & $4 l+2$ & $2 l+1$ \\
\hline 2 & $r-2^{*}$ & $4 l$ & $2 l$ \\
\hline 3 & $r-1^{*}$ & $4 l$ & $2 l$ \\
\hline
\end{tabular}

*(Residual simple.)

5.0. In this section we deal with equality in a Castelnuovo inequality when we have two linear series of different degrees, but of the same dimension. There are two natural questions to be asked here. The first one is, given a complete $g_{n}^{r}$ on a Riemann surface $X_{p}$, then $p$ has a maximum value $p_{1}$, when does the existence of a second linear series call it $h_{n+\rho}^{r}$ depress $p$ below the value $p_{1}$ ? The second question which we might pose is, given that $X_{p}$ admits a second linear series $h_{n+\rho}^{r}$, and supposing that $p$ attains its maximum value under these circumstances, are there models of such curves, and if there are, are they unique?

We give a partial answer to the first question. Suppose that $X_{p}$ admits a $g_{n}^{r}$ and that $p$ attains the maximum value allowed by the Castelnuovo inequality for one $g_{n}^{r}$. Let $\mathbf{K}$ denote the canonical series on $X_{p}$. We recall that if $n=(l+2) r-(l+t)$, where $1 \leq t \leq r-1$, then

$$
l g_{n}^{r}+g_{(r-t-1)(l+2)}^{r-t-1}=\mathbf{K}
$$

$X_{p}$ admits a $g_{l+\varepsilon+2}^{1}$ where $\varepsilon=0$ or 1 by 2.0 .6 . The presence of the linear series $h_{n+l+\varepsilon}^{r}=g_{n}^{r}+g_{l+2+\varepsilon}^{1}-\mathscr{P}-\mathscr{Q}$, where $\mathscr{P}$ and $\mathscr{Q}$ are two generic points of $X_{p}$ clearly does not depress the genus below the Castelnuovo lower bound. 
In the following sections we construct the answer to the second question and complete the answer to the first question posed above. In the remainder of this section we establish some notation and indicate the nature of our argument.

Let $\Psi(\alpha, \beta)$ equal the dimension of $\alpha g_{n}^{r}+\beta h_{n+\rho}^{r} \cdot{ }^{6}$ An elementary calculation shows that

$$
\Psi(\alpha, \beta)=\frac{(\alpha+\beta+1)(\alpha+\beta)}{2} r-\frac{(\alpha+\beta)(\alpha+\beta-1)}{2}+\alpha \beta .
$$

Let $\Delta(\alpha, \beta)$ denote the degree of $\alpha g_{n}^{r}+\beta h_{n+\rho}^{r}$. We note that $\Delta(\alpha, \beta)=$ $(\alpha+\beta) n+\beta \rho$, and that

$\Psi(\alpha-1, \beta+1)=\Psi(\alpha, \beta)+[\alpha-\beta-1]$ and $\Delta(\alpha-1, \beta+1)=\Delta(\alpha, \beta)+\rho$, and

$\Psi(\alpha+1, \beta-1)=\Psi(\alpha, \beta)+[\beta-\alpha-1] \quad$ and $\Delta(\alpha+1, \beta-1)=\Delta(\alpha, \beta)-\rho$.

If $\alpha g_{n}^{r}+\beta h_{n+\rho}^{r}$ is nonspecial, then the quantity $\Delta(\alpha, \beta)-\Psi(\alpha, \beta)$ corresponds to the genus of the curve $X_{p}$ assuming of course that $\alpha g_{n}^{r}+\beta h_{n+\rho}^{r}$ is complete.

Suppose that $\alpha g_{n}^{r}+\beta h_{n+\rho}^{r}$ is nonspecial and has minimal dimension, let $p(\alpha, \beta)=\Psi(\alpha, \beta)-\Delta(\alpha, \beta)$. Then $p(\alpha, \beta)$ is a quadratic function of $\alpha$ if we regard $\alpha+\beta$ as a fixed quantity. To locate the minimum value of $p(\alpha, \beta)$ - which will correspond to the genus of our curve - we only have to find the value of $\alpha$ so that

$$
p(\alpha+1, \beta-1) \geq p(\alpha, \beta) \leq p(\alpha-1, \beta+1) .
$$

Note that

$$
p(\alpha-1, \beta+1)=p(\alpha, \beta)+[\rho+\beta-\alpha+1]
$$

and that

$$
p(\alpha+1, \beta-1)=p(\alpha, \beta)+[\alpha-\beta-\rho+1] .
$$

Once we know that $\alpha g_{n}^{r}+\beta h_{n+\rho}^{r}$ is nonspecial, and we have calculated the upper bound on $p$, we get down to the task of establishing whether or not there is a model corresponding to $X_{p}$. As mentioned beforehand, the key to finding a model lies in producing a suitable $g_{T}^{1}$, and two divisors on $X_{p}, \mathscr{D}_{1}$ and $\mathscr{D}_{2}$, so that

We shall have

$$
h_{n+\rho}^{r}=g_{n}^{r}+g_{T}^{1}-\mathscr{D}_{1}-\mathscr{D}_{2}
$$

$$
(\alpha-1) g_{n}^{r}+\beta h_{n+\rho}^{r}+g_{m}^{\mu}=\mathbf{K} \quad \text { or } \quad \alpha g_{n}^{r}+(\beta-1) h_{n+\rho}^{r}+g_{m}^{\mu}=\mathbf{K},
$$

and we can extract some information about $g_{T}^{1}$, or about $\mathscr{D}_{1}$ and $\mathscr{D}_{2}$, from the residual series $g_{m}^{\mu}$. Specifically, $g_{m}^{\mu}$ will yield either the value of $T$, or it will give us a bound on the degree of $\mathscr{D}_{1}$. Once we have this information, we turn to a quadratic polynomial in two variables, $f(x, \varepsilon)$, to finish the job. Typically $x$ will be the degree of $\mathscr{D}_{1}$ and $T$ will be a function of $\varepsilon . f(x, \varepsilon)$ will have negative $x^{2}$ and $\varepsilon^{2}$ coefficents, and we show the maximum value of $f(x, \varepsilon)$ is equal to $p$, in which case we have a model, or less than $p$ in which case there is no model.

\footnotetext{
${ }^{6}$ We always assume that we can calculate this dimension by Accola's formula. Of course we are, at the same time, making a tacit assumption about $n, \alpha, \beta, \rho$, and $r$, namely, that $n+\rho \geq \alpha r+\beta(r-1)+1$. These are precisely the conditions that must hold for Accola's count to be valid.
} 
5.1. Let $n=(l+2) r-(l+t)$. Suppose that $X_{p}$, our curve, admits two linear series of the same dimension but of different degrees, $g_{n}^{r}$ and $h_{n+\rho}^{r}$. Following on our work in $\S 2$ we consider the following two possibilities for $n$ :

Case A: $n=\left(2 l_{0}+2\right) r-\left(l_{0}+t_{0}\right), l_{0} \geq 2$, where $0 \leq t_{0} \leq r-1$.

Case B: $n=\left(2 l_{0}+1\right) r-\left(l_{0}+t_{0}\right)$, where $0 \leq t_{0} \leq r-2$.

The two possibilities for $n$ may be described by $n=\left(2 l_{0}+1+\delta_{A}\right) r-\left(l_{0}+t_{0}\right)$. Where $0 \leq t_{0} \leq r-2+\delta_{A}$, and $\delta_{A}=1$ if we are in Case A and 0 if we are in Case B.

If $X_{p}$ admits two linear series of dimension $r$ and degree $n$, and $p$ attains its maximum value in the Castelnuovo inequality arising from these linear series, then from $\S 2$ with $j=2$,

$$
\left(l_{0}+\delta_{A}-1\right) g_{n}^{r}+l_{0} h_{n}^{r}+g_{m_{1}}^{\left(r-2+\delta_{A}-t_{0}\right)}=\mathbf{K} .
$$

Let the genus of $X_{p}$ in this case be denoted by $p_{2}$. Given a curve $X_{p}$ admitting two linear series of the same dimension but of different degrees, $g_{n}^{r}$ and $h_{n+\rho}^{r}$, where $p_{2} \leq p \leq p_{1}$, let $\mathbf{K}$ denote the canonical series for $X_{p}$, then we seek integers $\gamma$, and $\tau$, such that

$$
\left[l_{0}+\gamma\right] g_{n}^{r}+\left[l_{0}-\tau\right] h_{n+\rho}^{r}+g_{m}^{\mu}=\mathbf{K}
$$

for some $\mu$ and $m$.

There are four distinct cases to consider in both Case A and Case B they are the following:

(i) $\rho=2 \nu$ and $\nu=\lambda(2 r-1)+t_{0}-\Lambda$, where $0 \leq \Lambda \leq r-1$.

(ii) $\rho=2 \nu$ and $\nu=\lambda(2 r-1)+t_{0}-\Lambda$, where $r \leq \Lambda \leq 2 r-2$.

(iii) $\rho=2 \nu+1$ and $\nu=\lambda(2 r-1)+t_{0}-\Lambda$, where $r \leq \Lambda \leq 2 r-2$.

(iv) $\rho=2 \nu+1$ and $\nu=\lambda(2 r-1)+t_{0}-\Lambda$, where $0 \leq \Lambda \leq r-1$.

We shall indicate the argument for (i) in Case A. Afterwards, we tabulate the results for the other seven cases.

Case (i). $X_{p}$ admits a simple $g_{n}^{r}$ and $h_{n+2 \nu}^{r}$ where

$$
n=\left(2 l_{0}+2\right) r-\left(l_{0}+t_{0}\right), \quad \text { with } \nu=\lambda(2 r-1)+t_{0}-\Lambda \text {, for } 0 \leq \Lambda \leq r-1 \text {. }
$$

1. Calculating $p$. First, we calculate the Clifford index of $\left[l_{0}+\nu+\lambda\right] g_{n}^{r}+$ $\left[l_{0}-\nu+\lambda\right] h_{n+2 \nu}^{r}$. Let $\alpha=l_{0}+\lambda+\nu$, and $\beta=l_{0}+\lambda-\nu$. We denote by $C(\alpha, \beta)$ the Clifford index of $\alpha g_{n}^{r}+\beta h_{n+\rho}^{r}$. We find that

$$
C(\alpha, \beta)=(\alpha+\beta)(r-\Lambda-1) \text {. }
$$

In the case under consideration, $\left(2 l_{0}+2 \lambda\right)(r-\Lambda-1)$ is nonnegative. So we do not yet have an estimate for $p$. Next, we seek $C(\alpha+1, \beta)$. One can derive the formulae

$$
C(\alpha+1, \beta)-C(\alpha, \beta)=n-(2 \alpha+2 \beta+2) r+2 \alpha
$$

and

$$
C(\alpha, \beta+1)-C(\alpha, \beta)=n+\rho-(2 \alpha+2 \beta+2) r+2 \beta .
$$

Using the first result we find that

$$
C(\alpha+1, \beta)=-\left(2 l_{0}+2 \lambda+1\right) \Lambda-\left(l_{0}+\lambda-\nu\right)
$$


which is negative, therefore the linear series $W_{0}=(\alpha+1) g_{n}^{r}+\beta h_{n+2 \nu}^{r}$ is nonspecial. Therefore, we may use its dimension and degree to calculate the genus of our curve ${ }^{7}$. The degree of $W_{0}$ is $(\alpha+\beta+1) n+2 \nu \beta$, while the dimension is $(\alpha+\beta+1)(\alpha+\beta+2) r / 2-(\alpha+\beta+1)(\alpha+\beta) / 2+(\alpha+1) \beta$. We find that

$$
\begin{aligned}
p= & \frac{\left(2 l_{0}+2 \lambda+2\right)\left(2 l_{0}-2 \lambda+1\right)}{2} r+\lambda^{2}-l_{0}\left(l_{0}+1\right) \\
& -\nu\left(\nu-2 l_{0}-2 \lambda-1\right)-\left(2 l_{0}+2 \lambda+1\right) t_{0} .
\end{aligned}
$$

Thus, for suitable $\mu$, and $m$ we have

$$
\left[l_{0}+\nu+\lambda\right] g_{n}^{r}+\left[l_{0}-\nu+\lambda\right] h_{n+2 \nu}^{r}+g_{m}^{\mu}=\mathbf{K} .
$$

2. The residual series. As before, we calculate $\mu$, the dimension of the residual series from the formula $\mu=n-\theta_{0}-1$, where $\theta_{0}$ is the number of conditions that $g_{n}^{r}$ imposes on $W_{0}$. By sharpness, $\theta_{0}=\left(2 l_{0}+2 \lambda+1\right) r-\left(l_{0}+\right.$ $\lambda+\nu+1)+1$, we find that $\mu=r-\Lambda-1$. Since $0 \leq \Lambda \leq r-1,0 \leq \mu \leq r-1$. A calculation shows that

$$
m=\left(2 l_{0}+2 \lambda+2\right)(r-\Lambda-1) .
$$

Next, we calculate the dimension, $\mu_{1}$, of $g_{n}^{r}+g_{m}^{\mu}$. Using the formula derived in $\S 2, \mu_{1}=n+\mu-\theta_{1}$, where $\theta_{1}$ is the number of conditions that $g_{n}^{r}$ imposes upon $\mathbf{K}-g_{m}^{\mu}$. Since everything is sharp in the formula for the dimension of the preceding linear series, we find that $\theta_{1}=\left(2 l_{0}+2 \lambda\right) r-\left(l_{0}+\lambda+\nu\right)+1$. Substituting this value, and the expression for $n$, into our formula for $\mu_{1}$ we find that

$$
\mu_{1}=r+2 \mu \text {. }
$$

Finally, we compute the dimension, $\mu_{2}$, of $h_{n+2 \nu}^{r}+g_{m}^{\mu}$. It equals $n+\mu+2 \nu-\theta_{2}$ where $\theta_{2}$ is the number of conditions that $h_{n+2 \nu}^{r}$ imposes upon $\mathbf{K}-g_{m}^{\mu}$. By sharpness, $\theta_{2}=\left(2 l_{0}+2 \lambda\right) r-\left(l_{0}+\lambda-\nu\right)+1$, which gives

$$
\mu_{2}=r+2 \mu \text {. }
$$

3. The models. From 1.1.4 we see that if $\mu \leq r-3$ then $g_{m}^{\mu}$ is composite, if $\mu=r-2$ or $\mu=r-1$ then $g_{m}^{\mu}$ may be composite or simple. We investigate all of the possible cases below.

(a) Suppose that $g_{m}^{\mu}$ is composite. Then, $g_{m}^{\mu}$ is composed of a $g_{2 l_{0}+2 \lambda+2}^{1}$ which imposes two conditions on $g_{n}^{r}$. From 1.2.1

$$
h_{n+2 \nu}^{r}=g_{n}^{r}+g_{2 l_{0}+2 \lambda+2}^{1}-\mathscr{D}_{1}-\mathscr{D}_{2},
$$

where $\mathscr{D}_{1}+\mathscr{D}_{2}$ has degree $2 l_{0}+2 \lambda-2 \nu+2$. Since $g_{m}^{\mu}$ has no fixed points, one of $\mathscr{D}_{1}$ and $\mathscr{D}_{2}$ must have degree at least $l_{0}+\lambda-\nu+1$. Making use of our results from $\S 3$ we use $g_{n}^{r}$ to provide a map $\varphi: X_{p} \rightarrow Y$ where $Y$ is a rational normal surface scroll in $\mathbf{P}^{r}$. Recall that if $C \in|T H+\beta L|$ is smooth then

$$
p(C)=\frac{T[T-1](r-1)}{2}+[T-1][\beta-1] .
$$

In the situation under consideration, the image of $X_{p}$ under $\varphi$ will not be smooth since both $\mathscr{D}_{1}$ and $\mathscr{D}_{2}$ impose one condition on $g_{n}^{r}$. Let $x$ be the

\footnotetext{
${ }^{7} p(\alpha+2, \beta-1)=p(\alpha, \beta)+[2 \nu-2 \nu+2]$ and $p(\alpha, \beta+1)=p(\alpha, \beta)+[2 \nu-2 \nu]$
} 
degree of $\mathscr{D}_{1}$. Then we have $p \leq f(x)$ where

$$
\begin{aligned}
f(x)= & \frac{\left(2 l_{0}+2 \lambda+2\right)\left(2 l_{0}+2 \lambda+1\right)}{2}(r-1) \\
& +\left(2 l_{0}+2 \lambda+1\right)\left(n-\left(2 l_{0}+2 \lambda+2\right)(r-1)-1\right) \\
- & \frac{x(x-1)}{2}-\frac{\left(2 l_{0}+2 \lambda+2-2 \nu-x\right)\left(2 l_{0}+2 \lambda+1-2 \nu-x\right)}{2} .
\end{aligned}
$$

$f(x)$ attains its maximum at $\hat{x}=l_{0}+\lambda-\nu+1$. Evaluating, one finds that $f(\hat{x})=p$. We see, therefore, that $\mathscr{D}_{1}$ and $\mathscr{D}_{2}$ have the same degree. A plane model for $X_{p}$ can now be produced by projecting into $\mathbf{P}^{2}$ and the resulting curve (possibly after some quadratic tranformations, cf. §4) will have three ordinary singular points.

(b) We see from (5.1) and (5.2) that $g_{m}^{\mu}$ cannot be simple if $\mu=r-2$, if it were, then $X_{p}$ would admit a $g_{n-m}^{1}$ and a $g_{n+2 \nu-m}^{1}$, both of which impose two conditions on $g_{n}^{r}$, which is impossible.

(c) If $\mu=r-1$, and $g_{m}^{\mu}$ is simple, then $X_{p}$ admits a $g_{2 l_{0}+2 \lambda+\varepsilon+2}^{1}$. There exists a divisor $\mathscr{D}$ of degree $n-m$ imposing one condition on $g_{n}^{r}$ so that $g_{n}^{r}=g_{m}^{r-1}+\mathscr{D}$. Since $\mu_{2}=3 r-2$, we can deduce by the same argument as we used in 2.1.3 that $h_{n+2 \nu}^{r}=g_{n}^{r}+g_{T}^{1}-\mathscr{D}-\mathscr{D}_{1}$. Now, $n-m=l_{0}+\lambda-\nu+2$ and so, $p \leq f(\varepsilon)$, where $f(\varepsilon)$ is the usual quadratic expression (cf. (3.4)). In this case $f(\varepsilon)$ simplifies out to

$$
p-1-\frac{r}{2} \varepsilon^{2}+\frac{r+2}{2} \varepsilon
$$

We conclude that if $\varepsilon=1$, there is a model for $X_{p}$ in $\mathbf{P}^{r}$ admitting a $g_{2 l_{0}+2 \lambda+3}^{1}$, and possessing two singular points of multiplicity $l_{0}+\lambda+\nu+2$ and $l_{0}+\lambda-\nu+1$. This concludes our analysis of the the first case.

5.2. The calculations performed in the first case are typical of the ones for the other cases. The only subtlety required is when we have to deal with the cases $\mu=r-1$ or $\mu=r-2$, and the residual series may be simple or composite. In every case $X_{p}$ admits a model where $g_{m}^{\mu}$ is composite. When $g_{m}^{\mu}$ is simple there may not be a model. In these cases, calculating $\mu_{2}$ (the dimension of $\left.h_{n+\rho}^{r}+g_{m}^{\mu}\right)$ is usually the key to deciding whether or not a model may exist ${ }^{8}$. In Tables 5.2.1 and 5.2.2 we have marked with an asterisk the entries corresponding to a simple residual. The reader will notice, that in all but one case, it is possible to have a simple residual. Now we tabulate the result of our calculations for all possible cases. In each case the models exhibited can be (and have been) shown to be unique. In our table we use the following notation:

(i) $\nu=\lambda(2 r-1)+t_{0}-\Lambda$ where $0 \leq \Lambda \leq r-1$ or $r \leq \Lambda \leq 2 r-2$.

(ii) $\alpha$ and $\beta$ will be the integers which are functions of $\rho$ such that $\alpha g_{n}^{r}+$ $\beta h_{n+\rho}^{r}$ will have negative Clifford index. Once we have establised $\alpha$ and $\beta$ we can obtain an upper bound on $p$.

(iii) $\mu$ will be the dimension of the linear series $g_{m}^{\mu}$ such that $\hat{\alpha} g_{n}^{r}+\hat{\beta} h_{n+\rho}^{r}+$ $g_{m}^{\mu}=\mathbf{K}$ where $\{\hat{\alpha}, \hat{\beta}\}=\{\alpha-1, \beta\}$ or $\{\hat{\alpha}, \hat{\beta}\}=\{\alpha, \beta-1\}$.

(iv) $T$ will be the degree of the unique $g_{T}^{1}$ imposing two conditions on $g_{n}^{r}$ and $h_{n+\rho}^{r} T=\alpha+\beta+1$ or $\alpha+\beta+2$.

(v) $S=\max \left\{\operatorname{deg} \mathscr{D}_{1}, \operatorname{deg} \mathscr{D}_{2}\right\}$ where $h_{n+\rho}^{r}=g_{n}^{r}+g_{T}^{1}-\mathscr{D}_{1}-\mathscr{D}_{2}$.

\footnotetext{
${ }^{8}$ For example in (b) above, since $\mu_{2}=r+2 \mu, g_{m}^{r-2}$ could not be simple.
} 


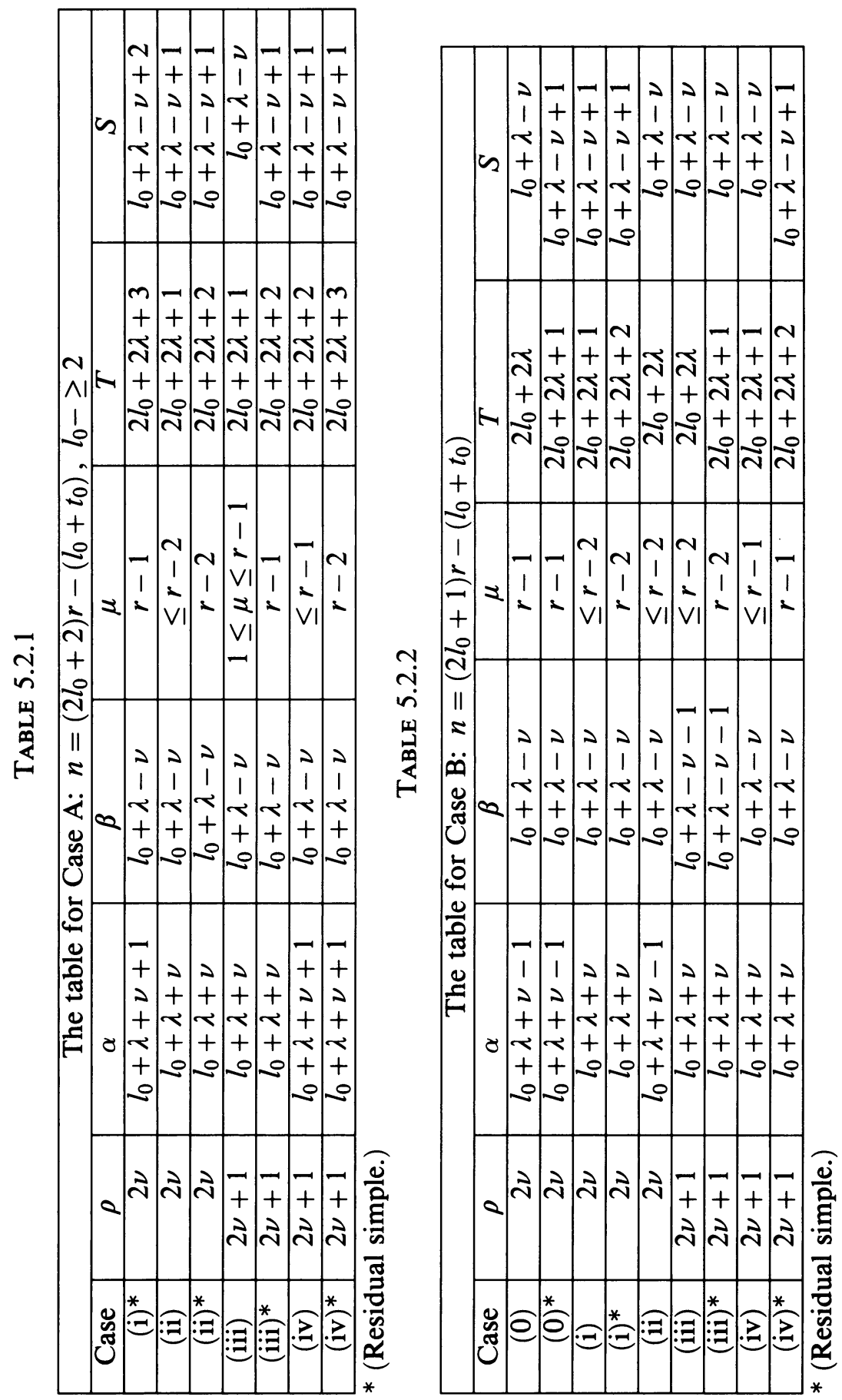


For a given $n$ and $r$ we are in Case A or B. Given $\rho$, we find $\alpha$ and $\beta$ so that $\alpha g_{n}^{r}+\beta h_{n+\rho}^{r}$ is nonspecial. Then we can calculate $p$. Next, we find $\mu$. Once this is determined we can find $T$, and the degrees of $\mathscr{D}_{1}$ and $\mathscr{D}_{2}$ are determined since they can differ by at most 1 and add up to $T-\rho$. Finally, the genus $X_{p}$ of our Riemann surface can be calculated from the formula

$$
\begin{aligned}
p= & \frac{T[T-1]}{2}(r-1)+[T-1][n-T(r-1)-1] \\
& -\frac{S[S-1]}{2}-\frac{[T-S-\rho][T-S-\rho-1]}{2},
\end{aligned}
$$

and the result of this calculation agrees with the upper bound in (ii). We also note that the arguments in $\S 4$ show that plane models for such curves can be constructed.

Remark 5.2.3. To see that $l$ (or $l+1$ ) is an upper bound on $\rho$ one just has to examine the tables. Setting $\beta=0$ or $\beta=1$ (depending on how we calculate $p$ ) will leave us in the case of one $g_{n}^{r}$. and give $\rho=\alpha=l$ or $\rho=\alpha=l+1$.

We sum up our findings in this section in the following theorem.

Theorem 5.2.4. If $r \geq 6$ and $l \geq 4$ and $n=(l+2) r-(l+t)$. Let $p_{1}$ be the Castelnuovo upper bound for a curve admitting one $g_{n}^{r}$. There exist generalised Castelnuovo curves of genus $p \leq p_{1}$ admitting $a g_{n}^{r}$ and $h_{n+\rho}^{r} \quad \forall \rho, 1 \leq \rho \leq l$. If $t=1$ then there exists a generalised Castelnuovo curve of genus $p_{1}$ admitting a $h_{n+l+1}^{r}$.

\section{REFERENCES}

1. R. D. M. Accola, On Castelnuovo's inequality for algebraic curves, Part 1, Trans. Amer. Math. Soc. 251 (1979), 357-373.

2. E. Arbarello, M. Cornalba, P. Griffiths, and J. Harris, Geometry of algebraic curves, vol. 1, Springer-Verlag, Berlin and New York, 1985.

3. G. Castelnuovo, Sui multiple di una serie di grupa di punti appartenente ad una curve algebraica, Atti Accad. Sci. Torino 28 (Memorie scelte) (1893).

4. J. L. Coolidge, A treatise on algebraic plane curves, Oxford Univ. Press, 1931; Dover, 1959.

5. M. Coppens, On G. Marten's characterization of smooth plane curves, Bull. London Math. Soc. 20 (1988), 217-220.

6. _ـ Smooth curves possessing many linear systems $g_{n}^{1}$, Arch. Math. (Basel) 52 (1989).

7. M. Coppens and T. Kato, Pencils on smooth curves implying special plane models (to appear).

8. P. Griffiths and J. Harris, Principles of algebraic geometry, Wiley, New York, 1978.

9. G. Martens, Eine Charakterisierung glatter ebener Kurven, Arch. der Math. 41 (1983), 37-43.

10. R. J. Walker, Algebraic curves, Princeton Univ. Press, 1950; Dover, 1962.

Kevin Street College of Technology, Kevin Street, Dublin, Ireland

E-mail address: 1.donohoe@dit.ie 